\title{
Absolvent_innen der Akademie
}

Abschlussjahrgänge 2000-2018 Kurzbericht

Abteilung Qualitätsentwicklung 
]a[ akademie der bildenden künste wien

Qualitätsentwicklung

Dieser Bericht basiert auf der Studie

Studienerfahrungen, Tätigkeitsfelder und künstlerisches Selbstverständnis der Absolvent_innen der Akademie der bildenden Künste Wien (2000-2018), die 2019/20 vom

\section{Zentrum für Soziale Innovation}

im Auftrag der Akademie der bildenden Künste Wien durchgeführt wurde.

]a[ akademie der bildenden künste wien

Qualitätsentwicklung

Veronika Litschel

Augasse 2-6

A-1090 Wien

Tel.: +43 1 58816-2510

Web: www.akbild.ac.at

E-Mail: qe@akbild.ac.at

Wien, im November 2020 


\section{INHALT}

EXECUTIVE SUMMARY

1 EINLEITUNG

1.1 Zielsetzung der Studie

1.2 Aufbau des Berichts

1.3 Lesehinweise und Definitionen

2 UMSETZUNG DER STUDIE

2.1 Methodisches Vorgehen

2.2 Das Datensample - Rücklauf

3 ERWERBSITUATION IM JAHR 2018

3.1 Berufs- und Tätigkeitsfelder

3.2 Einkommen

3.3 Gestaltung der Erwerbsarbeit und Arbeitszufriedenheit

4 BERUFLICHE WEGE SEIT DEM STUDENABSCHLUSS

4.1 Übergang in die Berufswelt

4.2 Erfolge und Brüche

4.3 Faktoren beruflicher Integration

4.4 Berufliche Belastungsfaktoren

5 DAS STUDIUM AN DER AKADEMIE

38

5.1 Reflexion des Studiums

5.2 Vorbereitung auf den Einstieg ins studienbezogene Feld

5.3 Vermittelte Kompetenzen und Fähigkeiten

5.4 Wirkung von Praktika und Mobilität

5.5 Unterstützungsangebote der Akademie

5.6 Zwischenfazit:

6 RESÜMEE

7 ANHANG

7.1 Indikatoren im Einzelnen 


\section{ABBILDUNGSVERZEICHNIS}

Abbildung 1: $\quad$ Erwerbsarbeitsbereiche der Absolvent innen im Jahr 2018 nach Studienbezogenheit und Geschlecht (binär) in Prozent

Abbildung 2: Wichtigstes Einkommen der Befragten im Jahr 2018 nach Einkommensarten in Prozent

Abbildung 3: Nettojahreseinkommen der Absolvent_innen im Jahr 2018 nach Einkommensklassen und Geschlecht (binär) in Prozent

Abbildung 4: Verteilung der Jahresnettoeinkommen nach Einkommensklassen und Geschlecht (binär), Studienrichtungen Künstlerisches Lehramt und Bildende Kunst in Prozent

Abbildung 5: Durchschnittliche Wochenstunden in Erwerbsarbeit nach Geschlecht (binär) und Haushaltszusammensetzung

Abbildung 6: Belastungsfaktoren in Bezug auf die Erwerbssituation nach Geschlecht (binär), Mittelwerte der Belastungsstärke

Abbildung 7: Wichtige Faktoren der Arbeitszufriedenheit und deren Erfüllungsgrad in Prozent

Abbildung 8: Einschätzung des Übergangs in die Berufswelt nach Geschlecht (binär) in Prozent

Abbildung 9: Herausforderungen beim Übergang in die Berufswelt (Auswahl) nach Geschlecht (binär) in Prozent

Abbildung 10: Gründe für Brüche in der Erwerbsbiografie (Auswahl) nach Geschlecht (binär)

Abbildung 11: Einflussfaktoren der Integration in Erwerbstätigkeit

in einem studienbezogenen Feld nach eingeschätzter Einflussstärke, Mittelwerte

Abbildung 12

Belastungsfaktoren nach ausgewählten Studienrichtungen (Mittelwerte)

Betroffenheit von Diskriminierung nach Geschlecht in Prozent

Häufigkeit von Diskriminierungserfahrungen Betroffener nach Geschlecht, Mittelwerte

Häufigkeit von Diskriminierungserfahrungen Betroffener nach Geburtsort, Mittelwerte

Einschätzung der Vorbereitung auf den Einstieg in ein studienbezogenes Feld in Prozent

Abbildung 18: Gegenüberstellung: Wichtigkeit der vermittelten Kompetenzen und Wunsch nach mehr Förderung, Mittelwerte 


\section{TABELLENVERZEICHNIS}

Tabelle 1:

Tabelle 2:

Tabelle 3:

Tabelle 4:

Tabelle 5:

Tabelle A1:

Tabelle A2:

Tabelle A3:

Tabelle A4:

Tabelle A5:

Tabelle A6:
Rücklaufquote nach Geschlecht

Rücklaufquote nach Studienrichtungen 14

Interviewte Personen nach Studienrichtungen 15

Einkommensstreuung nach Studienrichtung 20

Mobilitätshindernisse in Bezug auf Auslandsaufenthalte während des Studiums nach Geschlecht (binär) in Prozent

Darstellung differenzierter Indikatoren zu Abbildung 7 (Arbeitszufriedenheit)

Darstellung differenzierter Indikatoren zu Abbildung 11 (Integration in Erwerbstätigkeit)

Darstellung differenzierter Indikatoren zu den Abbildungen 13 und 14 (Diskriminierung nach Geschlecht)

Darstellung differenzierter Indikatoren zu den Abbildungen 15 und 16 (Diskriminierung nach Geburtsort)

Darstellung differenzierter Indikatoren zu Abbildung 18 (Wichtigkeit der vermittelten Kompetenzen und Wunsch nach mehr Förderung)

Darstellung differenzierter Indikatoren zu Abbildung 20 (ausgewählte Unterstützungsbereiche) 


\section{EXECUTIVE SUMMARY}

\section{Aim and objective}

The aim of the study is to understand the impact of studies at the Academy of Fine Arts Vienna on graduates' career paths. The objective is a differentiated analysis of the career paths of graduates who obtained their diploma, bachelor or master's degree at the Academy of Fine Arts Vienna in the period 2000-2018.

Methodologically, the study combines a quantitative and a qualitative approach. The main part is based on an online survey among graduates who completed their studies between 2000 and 2018. The collected results have been interpreted with different analytical methods. In addition, biographical-narrative interviews with graduates have provided deep insights into the career paths of the graduates.

\section{Career paths and working life}

One main result is that the preparation of graduates for their professional life in the artistic field has been successful. Graduates are particularly equipped with skills and competences that help to tackle the challenges of independent and self-motivated work in the artistic field. Most graduates pursue a study-related career. They reflect positively on the role of the academy with regard to their career paths.

Graduates have obtained a wide range of additional further education, further studies or vocational training. Similar to all students in Austria, the majority of them have a stronger social background, the minority comes from a working class' milieu.

Working life is organised through various contractual arrangements, such as freelancers, marginal employment or short-time-contracts and the combination of self-employment and employment. On the one hand, this depends on the field of study completed and the occupation. Graduates of the study program Education in the Arts are mostly employed. Graduates of the study program Fine Arts or Architecture are rather self-employed, often with short-term contracts, on a project basis and accompanied by/with the need to work in additional jobs.

Precarious employment conditions are the greatest challenge for graduates. Overall, the income of two thirds of the respondents is below the average income in Austria (median income). Furthermore, a significant gender pay gap can be identified. The respondents attribute gender inequality to the traditional distribution of unpaid work and the area of care and reproductive work. Women, especially women with care responsibilities, face more and stronger barriers in accessing the study-related labour market than men. 


\section{Study time at the Academy of Fine Arts Vienna}

Regarding the study time at the Academy of Fine Arts Vienna, graduates emphasize the great creative freedom and the possibility of experimentation. The artistic work is described as free from exploitation constraints but combined with feedback and support. The close supervision relationship between lecturers and students is generally described as helpful and positive, although ambivalences of dependence and close ties to lecturers - in particular professors - who evaluate performance, are also reflected.

Graduates feel empowered to work independently in their disciplines. They have developed and continue to develop their own positioning in the field. In terms of achieving this, they clearly relate to their time at the university, which provided them with the fundamental knowledge and skills. In sum, artistic knowledge and practice, fellow students in the central artistic subject and the lecturers were important resources for their personal artistic development.

The artistic and socio-political discourse at the Academy is characterized by a critical examination of the capitalist mainstream and the hegemonic interpretation of society and art. Some interviewees tended to feel excluded from the clear orientation of the discourse. Another point that was critically reflected upon is that the academic discourse is far from reality of the less formalized art market and the exploitation constraints of one's own work performance in connection with gaining a livelihood.

In the survey and in the interviews, the graduates were also asked about possible improvements and the desire for more support and resources. Above all, suggestions for the expansion of support were made in the areas of practical implementation of projects, management and entrepreneurial knowledge and networks. This does not necessarily mean that the existing offerings of the Academy of Fine Arts Vienna are classified as insufficient or inadequate in these points. The desire for more support can also be seen as a sign of trust and recognition. It can be assumed that both readings are represented in the answers.

Moreover, a large proportion of the graduates emphasise the need to expand knowledge and available budgets on public funding and instruments to secure the livelihood of artists but also the possibility of exhibitions, the provision of studios and project funds are demanded. Further problems are the lack of transparency with regard to contract conditions and the general level of income and unequal payment in the specific labour market as well as the partial lack of transparency in fees.

Many graduates are members of formal networks and interest groups (e.g. VBKÖ, WKO, IG Bildende Kunst). Informal networks are considered an important resource for contacts and the initiation of projects. It is emphasized that being present at events and the like is exhausting, despite important. Respondents mainly describe those networks as beneficial that have been created in the course of their studies, in the joint implementation of projects or in other joint undertakings.

In a concluding observation, it can be stated that life and work in the artistic field is characterized by precarity. In order to survive in this professional field, graduates need a high degree of endurance and self-confidence. The average income is relatively low. The diverse combinations of employment types lead to challenges in terms of long-term social security. Nevertheless, the majority of graduates (around 80\%) are employed in a studyrelated field. 


\section{DIE ERGEBNISSE IM ÜBERBLICK}

\section{Zielsetzung der Studie}

Die Zielsetzung der Studie ist eine differenzierte Analyse der Karriereverläufe von jenen Absolvent_innen, die ihr Diplom, ihren Bachelor- oder Master Abschluss an der Akademie der bildenden Künste Wien im Zeitraum von 2000-2018 gemacht haben. Der Fokus liegt auf dem Impact, den ihr Studium auf ihre Karriereverläufe hatte. Der Bericht betrachtet die Perspektive der Absolvent_innen und ihre Selbsteinschätzung.

Methodisch intergiert die Studie einen quantitativen und einen qualitativen Ansatz. Der Hauptteil bezieht sich auf eine Onlinebefragung der Absolvent_innen, die ihr Studium zwischen 2000 und 2018 abgeschlossen haben. Die erhobenen Ergebnisse sind mit verschiedenen analytischen Verfahren interpretiert worden. Biografisch-narrative Interviews mit Absolvent_innen ermöglichen einen vertieften Einblick in die Karriereverläufe.

\section{Karriereschritte und berufliche Situation}

Ein Hauptergebnis ist, dass die Vorbereitung der Absolvent_innen auf ihr Berufsleben im künstlerischen Umfeld gut gelingt. Insbesondere wurden Skills und Kompetenzen für die Herausforderungen in Zusammenhang mit selbständiger und selbstmotivierter Arbeit im künstlerischen Umfeld vermittelt. Die meisten Absolvent_innen positionieren sich in einer studienbezogenen Berufstätigkeit bzw. Erwerbstätigkeit und reflektieren die Rolle der Akademie in Bezug auf ihre Karriereverläufe positiv.

Absolvent_innen haben vielfältige zusätzliche Weiterbildungen, weitere Studien oder Berufsausbildungen. Ähnlich der Gesamtheit der Studierenden in Österreich sind sie in der Mehrzahl mit einem höheren sozialen Hintergrund ausgestattet, die Minderheit kommt aus einem Arbeitermilieu.

Das Erwerbsarbeitsleben ist geprägt von vielseitigen Vertragsformen und der Kombination von selbständiger und unselbständiger Beschäftigung. Unterschiede zeigen sich in der abgeschlossenen Studienrichtung und im Beruf. Absolvent_innen des Künstlerischen Lehramts sind zum Großteil angestellt tätig. Absolvent_innen der Bildenden Kunst oder der Architektur arbeiten vermehrt selbständig, vielfach mit kurzzeitigen Verträgen, auf Projektbasis oder sind geringfügig beschäftigt.

Erwerbsarbeitsprekarität ist die größte Herausforderung für die Absolvent_innen. Insgesamt liegt das Einkommen von zwei Drittel der Befragten unter dem durchschnittlichen Einkommen in Österreich (Medianeinkommen). Darüber hinaus ist ein signifikanter Gender Pay Gap feststellbar. Die Befragten führen die geschlechtliche Segregation auf die traditionelle Verteilung von unbezahlter Arbeit im Bereich der Sorge- und Reproduktionsarbeit zurück. Frauen, insbesondere Frauen mit Betreuungspflichten sind mit einer Reihe zusätzlicher Hürden beim Zugang zum Arbeitsmarkt konfrontiert. 


\section{Das Studium an der Akademie}

In der Reflexion der Studienzeit an der Akademie betonen die Absolvent_innen den großen kreativen Freiraum und die Ermöglichung von Experimenten. Die künstlerische Arbeit wird als frei von Verwertungszwängen, aber verbunden mit Feedback und Unterstützung beschrieben. Das enge Betreuungsverhältnis zwischen Lehrpersonal und Studierenden wird generell als hilfreich und positiv eingeschätzt, obwohl auch Ambivalenzen der Abhängigkeit und der engen Bindung an Lehrpersonen, die Leistungen bewerten, reflektiert werden.

Die Absolvent_innen fühlen sich befähigt, unabhängig in ihren Disziplinen zu arbeiten. Sie entwickelten und entwickeln stetig ihre eigene Positionierung im Feld. Hier ziehen sie eine klare Verbindung zu ihrer Studienzeit, die ihnen die Voraussetzungen dafür vermitteln konnte. Vor allem der künstlerische Unterricht, die Studienkolleg_innen im zentralen künstlerischen Fach und die Lehrpersonen waren hier wichtige Ressourcen der Entwicklung.

Laut Rückmeldungen der Absolvent_innen ist der künstlerische und gesellschaftspolitische Diskurs an der Akademie von einer kritischen Auseinandersetzung mit dem kapitalistischen Mainstream und der hegemonialen Interpretation von Gesellschaft und Kunst geprägt. Einige Interviewte fühlten sich von der engen Ausrichtung des Diskurses tendenziell exkludiert. Ein weiterer Punkt wird in diesem Zusammenhang kritisch reflektiert: der akademische Diskurs wird als weit weg von der Realität des wenig formalisierten Kunstmarkts und der Verwertungszwänge der eigenen Arbeitsleistung und der Existenzsicherung empfunden.

In der Befragung und in den Interviews wurden die Absolvent_innen auch nach Verbesserungsmöglichkeiten und dem Wunsch nach mehr Unterstützung und Ressourcen befragt. Vor allem im Bereich der praktischen Umsetzung von Projekten, betriebswirtschaftlichen und unternehmerischen Kenntnissen sowie dem Aufbau von Netzwerken kamen Vorschläge für den Ausbau von Unterstützungsangeboten. Das bedeutet nicht, dass es in diesen Bereichen keine Aktivitäten gab oder gibt, sondern spiegelt viel mehr die Wichtigkeit der einzelnen Ressourcen wider.

Ein Großteil der Absolvent_innen betont die Notwendigkeit des Ausbaus von öffentlicher Finanzierung und Instrumenten der Existenzsicherung für Künstler_innen. Aber auch die Möglichkeit von Ausstellungen, das Zur-Verfügung-Stellen von Ateliers und Projektmitteln wird eingefordert. Eine weitere Problematik ist die mangelnde Transparenz in Bezug auf Vertragskonditionen, die generell niedrigen Einkommen und die ungleiche Bezahlung sowie die teilweise Undurchsichtigkeit bei Honorarsätzen.

Viele Absolvent_innen sind Mitglieder in formalen Netzwerken und Interessensvertretungen (z.B. VBKÖ, WKO, IG Bildende Kunst). Informelle Netzwerke werden als wichtige Ressource für Kontakte und das Anbahnen von Projekten angesehen. Dabei betonen die Interviewten auf der einen Seite, dass die Präsenz bei Events und Ähnlichem wichtig und anstrengend ist. Auf der anderen Seite beschreiben die Befragten hauptsächlich jene Netzwerke als weiterbringend, die im Rahmen des Studiums, in der gemeinsamen Durchführung von Projekten oder bei anderen gemeinsamen Vorhaben entstanden sind.

Zusammenfassend kann festgehalten werden, dass das Leben und Arbeiten im künstlerischen Umfeld von Prekarität geprägt sind. Um in diesem beruflichen Feld zu bestehen, brauchen Absolvent_innen ein hohes Maß an Durchhaltevermögen und Selbstvertrauen. Das Durchschnittseinkommen ist vergleichsweise niedrig. Die vielfältigen Kombinationen von Beschäftigungsarten führen zu Herausforderungen in Bezug auf eine dauerhafte soziale Absicherung Trotzdem ist der überwiegende Teil der Absolvent_innen (rund 80\%) in einem studienbezogenen Feld erwerbstätig. 


\section{EINLEITUNG}

\subsection{Zielsetzung der Studie}

Die Akademie der bildenden Künste Wien hat Anfang 2019 eine Studie mit dem Ziel einer differenzierten Analyse der vielfältigen beruflichen Wege der Absolvent_innen beauftragt. Untersucht wurden ehemalige Studierende, die in den Jahren 2000-2018 ihr Diplom-, Bachelor- oder Masterstudium abgeschlossen haben. Dabei sollte im Forschungsansatz eine Verlaufsperspektive eingenommen werden. Der Impact des Studiums auf den beruflichen Werdegang der Absolvent_innen sollte besonders fokussiert werden. Im Zentrum der Erhebungs- und Analyseschritte standen folgende Fragestellungen:

$\neg \quad$ In welchen Berufs- und Tätigkeitsfeldern waren und sind Absolvent_innen der Akademie beschäftigt? Wie war und ist die Beschäftigungs- und Erwerbssituation? Welche biografischen Entwicklungen bzw. Brüche sind ablesbar?

$\neg \quad$ Was waren die wesentlichen Karriereschritte oder Stolpersteine im beruflichen Werdegang der Absolvent_innen und auf welche Faktoren sind diese rückführbar? Welche Bezüge können zur Akademie hergestellt werden?

$\neg \quad$ Welche Verbindungslinien zeigen sich zwischen Studium (z.B. Projektarbeiten, Praktika, Erwerbsarbeit, Qualifikationsprofil, Auslandsaufenthalten) und dem beruflichen Werdegang?

$\neg \quad$ Inwieweit haben soziale Faktoren (wie z.B. Geschlecht, Herkunft, familiärer Hintergrund) den Studienverlauf und den beruflichen Weg beeinflusst? Haben hier spezifische Einflussgrößen der Akademie während des Studiums oder im späteren beruflichen Leben fördernd oder unterstützend eingehakt?

Das Projektdesign stellt die Selbsteinschätzung der Absolvent_innen in den Mittelpunkt. Die Erhebungen und die entsprechenden Interpretationen spiegeln damit die Sichtweise der Befragten sowie ihre persönliche Bewertung des Studiums und ihrer beruflichen Situation wider.

\subsection{Aufbau des Berichts}

Der vorliegende Bericht basiert auf der ZSI-Absolvent_innen-Studie 2000-2018 ${ }^{1}$. Die Abteilung Qualitätsentwicklung der Akademie hat in redaktioneller Arbeit die aussagekräftigen Ergebnisse interpretiert und aufbereitet.

Der vorliegende Bericht gliedert sich in folgende Abschnitte:

$\neg \quad$ Umsetzung der Studie

$\neg$ Erwerbssituation im Jahr 2018

$\neg$ Berufliche Wege seit dem Studienabschluss

$\neg \quad$ Das Studium an der Akademie

$\neg$ Resümee

1 Barbara Glinsner, Ursula Holtgrewe, Irina Vana (Zentrum für soziale Innovation - ZSI: (2020): Studienerfahrungen, Tätigkeitsfelder und künstlerisches Selbstverständnis der Absolvent_innen der Akademie der bildenden Künste Wien (2000-2018). Studie im Auftrag der Akademie der bildenden Künste Wien. Nachfolgend: „ZSI Absolvent_innenstudie 2000-2018“" 
Im ersten Abschnitt - Umsetzung der Studie - werden das methodische Vorgehen sowie die Untersuchungsgruppe erläuternd vorgestellt, um darzulegen, wie die Ergebnisse und Trends der Absolvent_innen-Studie gewonnen wurden.

Abschnitt 2 befasst sich mit der Erwerbssituation der befragten Absolvent_innen im Jahr 2018. Insbesondere die Berufs- und Tätigkeitsfelder stehen im Fokus. Betrachtet werden die Erwerbs- und Einkommenssituation, die Arbeitszufriedenheit sowie die Einschätzung der eigenen Etablierung im Feld.

Der dritte Abschnitt zeigt die Verläufe in Bezug auf die Berufs- und Tätigkeitsfelder seit dem Studienabschluss auf. Daraus gehen Informationen über Strategien und Wege, die Übergänge nach dem Studium zu bewältigen, hervor. Die erhobenen Daten bzw. deren vorliegende Auswertungen zeigen unterstützende und hinderliche Faktoren aus der Sicht der Befragten auf.

Die Einschätzung des Studiums an der Akademie sowie dessen Einfluss auf die berufliche und persönliche Entwicklung sind Inhalt des vierten Abschnitts, der mit „Das Studium an der Akademie" übertitelt ist. Dabei werden neben erworbenen Qualifikationen und Kompetenzen sowie der Rolle von berufsspezifischen Netzwerken und dem Zugang zu diesen auch Fragen in Richtung einer Entwicklungsperspektive der Akademie untersucht.

Das abschließende Resümee fasst die Ergebnisse mit dem Fokus auf die Rolle der Akademie der bildenden Künste Wien aus Sicht ihrer Absolvent_innen zusammen. Dies kann als Input für die stetige Debatte um die Weiterentwicklung der Akademie und ihrer Studien oder Unterstützungsangebote dienen.

\subsection{Lesehinweise und Definitionen}

Um die Anonymität der befragten Absolvent_innen zu wahren, bzw. wenn Rückschlüsse auf konkrete Personen gezogen werden könnten, werden Ergebnisse mit kleinen Fallzahlen $^{2}$ nicht oder lediglich als Trends im Fließtext dargestellt.

In der Regel werden die Auswertungen für das Gesamtsample - also für alle Befragten dargestellt. Gibt es aussagekräftige Abweichungen nach Studienrichtungen oder nach soziodemografischen Merkmalen, wird darauf separat eingegangen.

Ebenfalls zur Wahrung der Anonymität werden bei Auswertungen nach Geschlecht Anteile von Befragten, die sich nicht-binär zuordnen, nicht generell ausgewiesen. Soweit sie von der Grundgesamtheit abweichen, wird jedoch im erläuternden Text auf ihre spezifische Situation eingegangen.

Aus Gründen der Lesbarkeit wurden bei verschiedenen Einschätzungsfragen die einzelnen Antworten in thematische Gruppen zusammengefasst. Welche Fragestellungen dort jeweils subsumiert sind, ist in Fußnoten erklärt. Im Anhang finden sich Tabellen, in denen jede Antwortmöglichkeit und die dazugehörigen Verteilungen bzw. die Mittelwerte differenziert dargestellt sind.

2 Bei Erhebungen und Ergebnisdarstellungen ist aus forschungsethischer Sicht die Anonymität der Befragten zu wahren. Statistik Austria veröffentlicht beispielsweise generell keine Daten, die auf Fallzahlen mit weniger als 30 Personen beruhen. Im Rahmen dieses Berichts wird etwas flexibler vorgegangen. Anleitend ist bei jeder Darstellung die Überprüfung, ob Rückschlüsse auf konkrete Personen gezogen werden können. 
Gängige Begrifflichkeiten rund um die Thematik der Arbeit, der künstlerischen Tätigkeit oder auch des Arbeitsmarkts sind auch in der wissenschaftlichen Literatur nicht immer trennscharf definiert. Im Rahmen der Studie und des Berichts werden folgende Definitionen verwendet:

$\neg \quad$ Mit Erwerbsarbeit oder Erwerbstätigkeit werden entlohnte Tätigkeiten in selbstständiger oder unselbständiger Beschäftigung gefasst, die vertraglich festgelegt sind.

$\neg$ Unselbständige Erwerbsarbeit beschreibt Berufstätigkeit im Rahmen einer Anstellung mit bestehendem Arbeitsvertrag, unabhängig von der konkreten Ausgestaltung der Arbeitszeit (Vollzeit, Teilzeit, geringfügig).

$\neg$ Selbständige Erwerbsarbeit wird in einer breiten Definition verwendet. Darunter fallen Werkverträge mit und ohne angemeldetem Gewerbe, Unternehmer_innen als Einzelunternehmen oder mit Angestellten, freie Dienstverträge ${ }^{3}$, freiberufliche Honorartätigkeit und vieles mehr. In all diesen Konstellationen sind sowohl steuerrechtliche als auch sozialversicherungstechnische Abgaben und Vorgaben durch die Auftragnehmer_innen selbst zu organisieren.

$\neg$ Künstlerische Arbeit ist - in Abgrenzung zu sozialversicherungsrechtlich definierter Erwerbarbeit - mit beruflicher Arbeit im künstlerischen Umfeld beschrieben, die projektförmigen und nicht im Rahmen von vertraglich fixierten Aufträgen geleistet wird, deren finanzielle Verwertbarkeit damit zwar durchaus geplant sein kann, aber noch nicht sichergestellt ist. In diesem Sinne wäre z.B. die Herstellung eines künstlerischen Werks, das ohne konkreten Auftrag, aber mit der Absicht es zu verkaufen, ebenso künstlerische Arbeit wie die Beteiligung an Projektausschreibungen oder Wettbewerben.

$\neg$ Künstlerische Tätigkeit wird als ein Überbegriff für unterschiedliche Formen der Arbeit im künstlerischen Umfeld, unabhängig von Entlohnung und Vertragsform verwendet.

$\neg$ Studienbezogen fasst jene Erwerbsarbeit oder Tätigkeit, die von den Befragten als mit ihrem Studium oder ihrer Studienrichtung verbunden eingeschätzt wird. Die Definition ist weit gefasst.

3 Freie Dienstverträge im künstlerischen Bereich stellen eine Sonderregelung dar und gelten als „Neue Selbständige“, die der gewerblichen Sozialversicherung unterliegen. Dies weicht von anderen Regelungen der freien Dienstverträge ab. Beschäftigung in Form von Freien Dienstverhältnissen in anderen Bereichen wird subsumiert, da die Abgrenzung komplex und im Rahmen einer Befragung nur sehr aufwendig zu erheben ist. Der Erkenntniswert steht in diesem Fall in keinem Verhältnis zum Erhebungsaufwand. 


\section{UMSETZUNG DER STUDIE}

\subsection{Methodisches Vorgehen}

Die Absolvent_innenstudie 2000-2018 wurde im Mixed-Method-Verfahren durchgeführt. Dabei werden quantitative und qualitative Erhebungsmethoden angewandt und in einer integrierten bzw. aufeinander bezogenen Form ausgewertet. Eine Online-Erhebung mit einem umfassenden Fragebogen ist die Grundlage für die quantitativen Daten. Biografischnarrative Interviews als qualitative Erhebungsmethode geben exemplarische vertiefte Einblicke. Alle im vorliegenden Bericht angeführten Zitate stammen aus diesen Interviews.

Die Erhebungs- und Auswertungsarbeiten wurden von der sozialwissenschaftlichen Forschungseinrichtung Zentrum für soziale Innovation (ZSI) in Zusammenarbeit mit der Akademie durchgeführt.

Der Fragebogen für die Generierung quantitativer Daten umfasst die folgenden sechs Themenbereiche:

$\neg \quad$ Studium und Ausbildung

$\neg$ Berufs- und Erwerbseinstieg

$\neg$ beruflicher Werdegang

$\neg$ Tätigkeitsbereiche und Arbeitssituation im Jahr 2018

$\neg$ Netzwerke

$\neg$ soziodemografische Daten

Über den Alumnae_Alumni-Verein der Akademie konnte Kontakt zu den dort gemeldeten Absolvent_innen der Abschlussjahrgänge 2000 bis 2018 aufgenommen werden. Darüber hinaus gingen über einschlägige Netzwerke, wie z.B. die IG Bildende Kunst, Einladungen zur Teilnahme an der Online-Befragung ins Feld.

\subsection{Das Datensample - Rücklauf}

Die Grundgesamtheit der zu Verfügung stehenden Kontaktadressen lag bei 2.092 potentiellen Teilnehmer_innen. Diese haben sowohl eine Information der Akademie über die Studie und deren Zielsetzungen der Befragung als auch vom ZSI einen persönlichen Link zum Fragebogen erhalten. 529 Fragebögen wurden ausgefüllt und retourniert. Dies entspricht einer Rücklaufquote ${ }^{4}$ von $26 \%$. Dies liegt in der erwartbaren Bandbreite von OnlineBefragungen und kann als zufriedenstellend gewertet werden. 
Tabelle 1

Rücklaufquote der Befragung nach Geschlecht

\begin{tabular}{lr} 
Geschlecht & Anteile in Prozent \\
\hline weiblich & $58 \%$ \\
männlich & $36 \%$ \\
divers & $1 \%$ \\
keine Angabe zum Geschlecht & $5 \%$ \\
\hline Gesamt & $\mathbf{1 0 0 \%}$ \\
\hline Anzahl Personen & $\mathbf{5 2 9}$
\end{tabular}

Quelle: ZSI - Absolvent_innenstudie 2000-2018

Die Verteilung nach Frauen und Männern im Befragungs-Sample entspricht der Zusammensetzung in den Registerdaten nach der Universitäts-Studienevidenzverordnung ${ }^{5}$, die alle Studierenden umfasst. Dort ist die binäre Geschlechterverteilung ${ }^{6}$ der Studierenden an der Akademie der bildenden Künste Wien für den Betrachtungszeitraum mit 60\% Frauen und 40\% Männern ausgewiesen.

In der Untersuchungsgruppe sind Absolvent_innen aller Studienrichtungen der Diplom-, Bachelor- und Masterstudien der Akademie vertreten. Die Verteilung der Befragten nach Studienrichtungen entspricht weitgehend der Verteilung der belegten Studien an der Akademie. Acht Befragte haben angegeben mehr als ein Studium im Betrachtungszeitraum an der Akademie der bildenden Künste Wien absolviert zu haben.

Tabelle 2

Rücklaufquote der Befragung nach Studienrichtungen (Mehrfachantworten möglich)

\begin{tabular}{lrr} 
Studienrichtungen & absolvierte Studien & Anteile in Prozent \\
\hline Bildende Kunst & 320 & $60 \%$ \\
Künstlerisches Lehramt & 104 & $19 \%$ \\
Architektur & 49 & $9 \%$ \\
Konservierung und Restaurierung & 31 & $6 \%$ \\
Bühnengestaltung & 21 & $4 \%$ \\
Master in Critical Studies & 12 & $2 \%$ \\
Gesamt & $\mathbf{5 3 7}$ & $\mathbf{1 0 0 \%}$
\end{tabular}

Quelle: ZSI - Absolvent_innenstudie 2000-2018

Das durchschnittliche Alter der befragten Absolvent_innen lag zum Befragungszeitpunkt bei 37 Jahren. Der Studienabschluss lag im Mittel sieben Jahre zurück. Jüngere Abschlussjahrgänge sind leicht überrepräsentiert. So haben rund $60 \%$ der Befragten ihr Studium in den Jahren 2010 bis 2018 beendet. Dementsprechend verteilen sich 40\% des Samples auf Studienabschlüsse der Jahre 2000 bis 2009. Ein Grund dafür ist, dass ältere Abschlussjahrgänge schwieriger über die bestehenden Kontaktdaten erreichbar sind.

Bei über der Hälfte der Personen im Sample verfügt mindestens ein Elternteil über einen akademischen Abschluss. Etwas über ein Drittel ist im Ausland geboren, der überwiegende Teil davon in einem Mitgliedsstaat der EU.

5 https://unidata.gv.at/Pages/auswertungen.aspx

6 Diese Datenquelle erfasst nur binäre Geschlechtszuordnungen. 
Das Datensample zeigt damit einen Querschnitt von Absolvent_innen, der weitgehend der Zusammensetzung der Studierenden an der Akademie der bildenden Künste Wien entspricht. Studienabschluss und Befragungszeitraum liegen im Mittel zeitlich ausreichend auseinander, um Erkenntnisse über Berufswege und Übergänge nach dem Studium zu erhalten.

\subsection{Zusammensetzung der Interviews}

Für die Akquise von Interviewpartner_innen wurden seitens des ZSI verschiedene Wege genutzt. Kontaktdaten sind über den Katalog von Abschlussarbeiten auf der Website der Akademie und ergänzende Recherchen in den Online-Portalen Linked-In und Google identifiziert worden. Darüber hinaus konnte im Fragebogen die Bereitschaft für ein Interview bekundet werden.

Bei der Auswahl der Interviewten waren die Repräsentanz aller Studienrichtungen der Akademie mit Ausnahme der Doktorate, die Geschlechterverteilung und die Herkunft anleitend. Insgesamt wurden 15 biografisch-narrative Interviews geführt.

Tabelle 3

Interviewte Personen nach Studienrichtung

$\begin{array}{lr}\text { Studienrichtung } & \text { Anzahl Interviews } \\ \text { Bildende Kunst } & 6 \\ \text { Künstlerisches Lehramt } & 3 \\ \text { Architektur } & 2 \\ \text { Konservierung und Restaurierung } & 1 \\ \text { Bühnengestaltung } & 2 \\ \text { Master in Critical Studies } & 1 \\ \text { Gesamt } & 15\end{array}$

Quelle: ZSI - Absolvent_innenstudie 2000-2018

Neun Interviewte ordnen sich dem weiblichen Geschlecht zu, fünf dem männlichen, eine Person ordnet sich nicht binär zu.

Die Interviews wurden wortgenau transkribiert und anschließend in einem kombinierten Verfahren von Kodierung und Sequenzanalyse ausgewertet, um einerseits mittels Kategorisierung über Kodes Muster aufzuzeigen und darüber hinaus latente Sinnstrukturen aufzudecken. Dadurch konnten Verläufe und Brüche sowie Bewältigungsstrategien aufgespürt und exemplarisch dargestellt werden. Wie in den quantitativen Erhebungen beruhen auch die Aussagen und (beruflichen) Kategorisierungen der Interviewten auf ihrer Selbsteinschätzung. 


\section{ERWERBSITUATION IM JAHR 2018}

\subsection{Berufs- und Tätigkeitsfelder}

Eine leitende Fragestellung der Studie war, wie sich die Berufs- und Tätigkeitsfelder der Absolvent_innen in Bezug zu ihrem Studium darstellen. Die Befragten wurden demnach gebeten, ihre derzeitige Erwerbstätigkeit danach einzuschätzen, ob diese in einem studienbezogenen Feld verortet sei. Die Fragestellungen beziehen sich auf die Situation der Absolvent_innen im Jahr 2018. Dieser Zeitraum liegt unmittelbar vor dem Erhebungszeitpunkt im Sommer 2019, dadurch kann ein möglichst aktueller Eindruck über die Lage der Absolvent_innen vermittelt werden.

Abbildung 1

Erwerbsarbeitsbereiche der Absolvent_innen im Jahr 2018 nach Studienbezogenheit und Geschlecht (binär) in Prozent

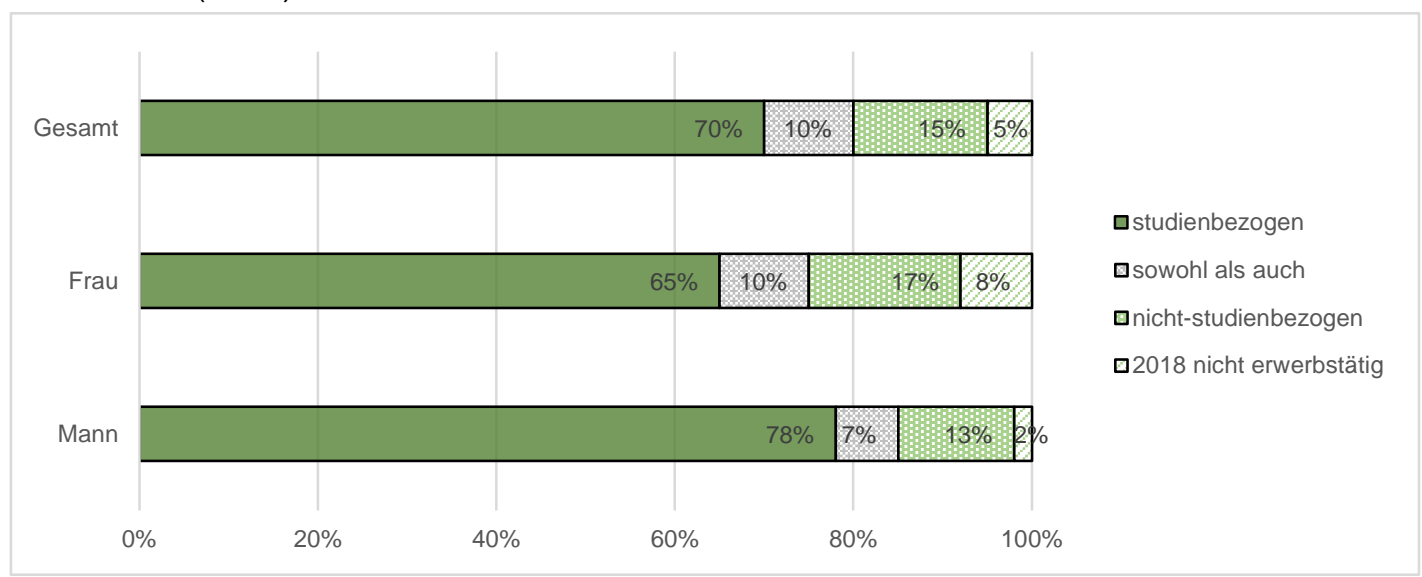

Quelle: ZSI - Absolvent_innenstudie 2000-2018

Ein Großteil der Befragten gibt an, einer Erwerbsarbeit nachzugehen, die in Bezug zu ihrem absolvierten Studium steht. Der Anteil der Frauen, die in einem studienbezogenen Bereich erwerbsarbeiten, ist niedriger als jener der Männer. Analog arbeiten sie zu einem höheren Anteil in einem anderen Bereich oder waren 2018 nicht erwerbstätig. Befragte, die sich keinem binären Geschlecht zugeordnet ${ }^{7}$ haben, geben zu $80 \%$ an im studienbezogenen Feld erwerbstätig zu sein. Damit erreichen sie Werte über den Männern, demgegenüber waren sie aber zu einem höheren Anteil als männliche Befragte im Jahr 2018 nicht erwerbstätig (7\%).

Studienbezogene Erwerbsarbeit wird durchaus breit verstanden. Es konnten vielfältige Zugänge herausgearbeitet werden. Zusammenfassend können drei Gruppen differenziert werden:

$\neg$ Erwerbstätigkeit fast ausschließlich im Feld des Studiums (z.B. arbeitet eine Absolventin der Bühnengestaltung als Bühnenbildnerin),

$\neg$ Erwerbstätigkeit in Anlehnung an das Studium durch Nutzung von dort erlangten Qualifikationen und Kompetenzen (z.B. ist eine Absolventin des Künstlerischen Lehramts als Selbständige in der Werbebranche und Modebloggerin tätig),

\footnotetext{
${ }^{7}$ Diese Personen haben sich entweder der Kategorie „divers“ zugeordnet oder die Angabe verweigert.
} 
$\neg$ Erwerbstätigkeit im Kulturbereich, um zusätzlich oder parallel zur studienbezogenen Arbeit ein ausreichendes Einkommen zu erzielen (z.B. ist ein Absolvent der Bildenden Kunst neben seiner künstlerischen Arbeit als Kunstvermittler tätig).

Auffällig ist, dass Absolvent_innen von Studienrichtungen, die mit einer formal benötigten Voraussetzung zur facheinschlägigen Berufsausübung abschließen, eher einer Erwerbsarbeit ausschließlich im Studienfeld nachgehen.

Dies gilt für die absolvierten Studien Künstlerisches Lehramt und Architektur. Ebenso ist in der Bühnengestaltung und der Konservierung und Restaurierung der Anteil jener, die sich eindeutig einem studienbezogenen Beruf zuordnen hoch.

In der Bildenden Kunst ist eine größere Vielfältigkeit feststellbar. Die Tätigkeitsfelder sind über viele Bereiche der Kulturarbeit und Kreativwirtschaft verstreut. Dabei wird Erwerbsarbeit im Kulturbereich oder in der Kreativwirtschaft mit dem Selbstverständnis künstlerischer Tätigkeit verbunden.

Diese Einschätzungen werden durch Aussagen in den Interviews verdeutlicht:

Mittlerweile bin ich ja seit 2003 selbständig als Grafikerin und da hab ich bei meinen Projekten schon sehr große künstlerische Freiheit. (Absolventin der Bildenden Kunst, Studienabschluss 2010)

Und dann fährt man vielleicht irgendwo hin und sammelt Geschichten und macht draus Features. Das ist eine wunderschöne Arbeit, die ja auch eine Art Kunst ist. (Absolventin der Bildenden Kunst, Studienabschluss 2011)

Die Verbindung der künstlerischen Tätigkeit ${ }^{8}$ mit „Jobs“, die nicht als eigentlicher Beruf angesehen werden, sondern der Existenzsicherung dienen und gleichzeitig ermöglichen, die eigene künstlerische Arbeit freier vom unmittelbaren Verwertungszwang auszuüben, ist eine verbreitete Form der studienbezogenen Erwerbsarbeit. Dabei ist den Interviewten wichtig, auch die „Jobs“ im künstlerischen oder kulturellen Umfeld anzusiedeln. Das reicht von Besuchs- und Aufsichtsdiensten in Museen, über das Eventmanagement und Marketing bis hin zur Kunstvermittlung.

Zusammenfassend kann damit festgehalten werden, dass ein abgeschlossenes Studium an der Akademie in der Regel zu einer Berufsausübung im künstlerischen Umfeld führt. 


\subsection{Einkommen}

Erwerbsarbeit stellt die Haupteinkommensquelle der Absolvent_innen im Jahr 2018 dar. Für diese Einschätzung konnten im Fragebogen verschiedene Einkommensarten ausgewählt und nach Wichtigkeit gereiht werden. Auf die Frage, welche Einkommensart im Jahr 2018 besonders wichtig war, gaben zwei Drittel der Befragten an, dass Erwerbsarbeit einen wichtigen Anteil ihres individuellen Einkommens ausmacht (Selbsteinschätzung).

Abbildung 2 zeigt die Verteilung der wichtigsten Einkommen nach Einkommensarten. Demnach ist für einen Anteil von $42 \%$ im Befragungssample unselbständige Erwerbsarbeit in Form einer Anstellung der wichtigste Bestandteil des Einkommens.

Abbildung 2

Wichtigstes Einkommen der Befragten im Jahr 2018 nach Einkommensarten in Prozent

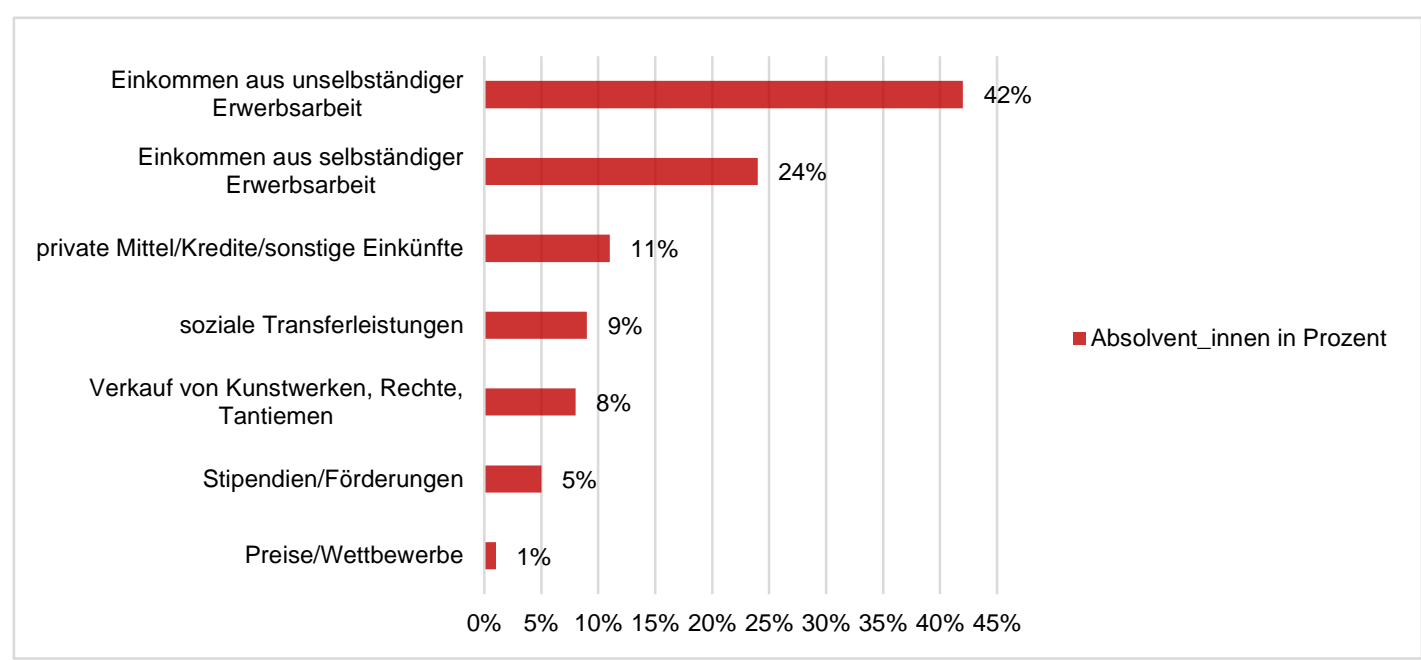

Quelle: ZSI - Absolvent_innenstudie 2000-2018

Die Ergebnisse der Erhebung zeigen, dass Einkommen aus verschiedenen Einkommensarten kombiniert werden. Die Einschätzung der Höhe des persönlichen Einkommens ist deshalb mit Unschärfen verbunden. Insbesondere bei selbständiger Erwerbsarbeit sind diese Angaben in Befragungen häufig ungenau. Um dem entgegenzuwirken, wurden für die Einschätzung der Einkommenshöhe im Fragebogen Einkommensklassen abgefragt und in der Auswertung auf Nettojahreseinkommen hochgerechnet. Die Verteilung ist in Abbildung 3 dargestellt.

Die Einkommenshöhe aller persönlichen Einkommen liegt für zwei Drittel der Befragten unter dem Medianeinkommen ${ }^{9}$ für einen Einpersonen-Haushalt in Österreich im Jahr 2018. Dieses lag laut Statistik Austria bei 25.175 Euro $^{10}$. Damit zeigt sich deutlich die Prekarität des künstlerischen Arbeitens.

9 Das Medianeinkommen gibt jenen Wert an, der genau in der Mitte aller Einkommen liegt. Dies ist für Verteilungsmaße eine exaktere Berechnungsweise als das Durchschnittseinkommen, da Abweichungen nach oben und nach unten nicht in den Wert eingehen.

$10 \mathrm{Vgl.:}$ https://www.statistik.at/web de/statistiken/menschen und gesellschaft/soziales/haushaltseinkommen/index.html. 
Abbildung 3

Nettojahreseinkommen der Absolvent_innen im Jahr 2018 nach Einkommensklassen und Geschlecht (binär) in Prozent

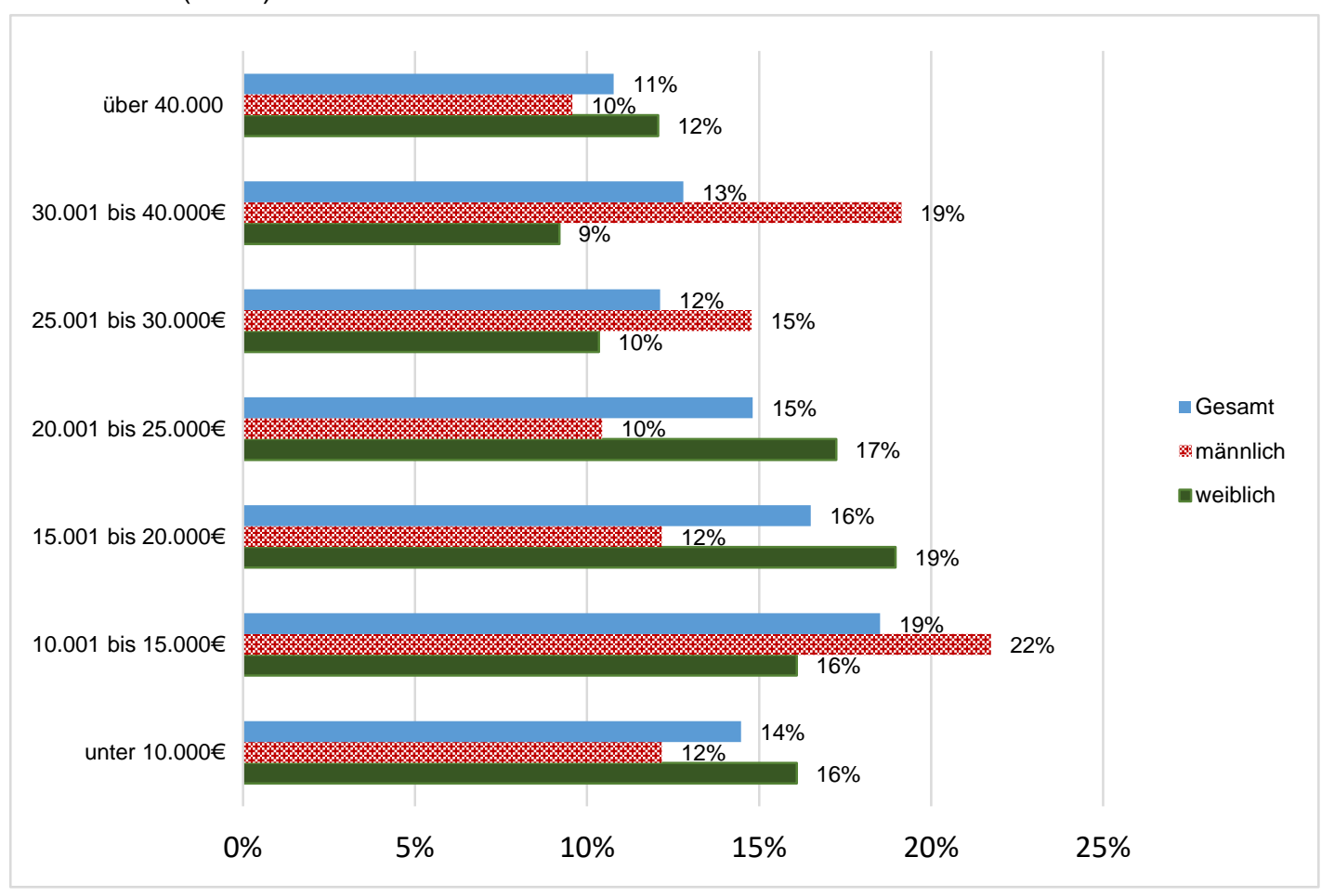

Quelle: ZSI - Absolvent_innenstudie 2000-2018, eigene Berechnungen

Die Einkommensverteilung nach Geschlecht (binär) zeigt, dass in den höheren Einkommensklassen stärker männliche Absolvent_innen vertreten sind als weibliche. Eine Ausnahme bildet die Einkommensklasse über 40.000 Euro jährlich, allerdings sind in dieser nur sehr wenige Personen vertreten. Die Darstellung differenziert nicht nach dem Beschäftigungsausmaß (Teilzeit- oder Vollzeitbeschäftigung), weil dies bei einem hohen Anteil an selbständiger Tätigkeit und von Kombinationen verschiedener Beschäftigungsarten auf Grundlage einer Befragung nicht valide durchgeführt werden kann.

Die untenstehende Abbildung 4 vergleicht die Nettojahreseinkommen nach Geschlecht (binär). Für die Illustration des Gender Pay Gaps wurden die Studienrichtungen Bildende Kunst und das Künstlerische Lehramt ausgewählt. Die erhobenen Daten machen für beide eine aussagekräftige Darstellung nach Geschlecht möglich.

Es zeigen sich auffällige Unterschiede in den Erwerbseinkommen. Im Künstlerischen Lehramt ist ein deutlicher Gender Pay Gap zu beobachten. In der untersten Einkommensklasse finden sich weder weibliche noch männliche Absolvent_innen dieses Studiums. Frauen sind allerdings in den beiden nächst höheren Einkommensklassen (Jahresnettoeinkommen von 10.001 bis 15.000 Euro bzw. von 15.001 bis 20.000 Euro) vertreten, während keiner der befragten Männer ein Einkommen der unteren drei Einkommensklassen angegeben hat. Alle Einkommensangaben von Männern, die ein Studium des Künstlerischen Lehramts absolviert haben, verteilen sich auf die höheren Einkommensklassen.

Da die Verdienststruktur im öffentlichen Dienst allerdings eine vergleichsweise geringe Spreizung nach dem Geschlecht aufweist, können als Gründe für die Differenz das Ausmaß der Erwerbsarbeit, eine Beschäftigung in einem anderen Berufsbereich sowie Kombinationen von Beschäftigungsbereichen angeführt werden. 
Abbildung 4

Verteilung der Jahresnettoeinkommen nach Einkommensklassen und Geschlecht (binär), Studienrichtungen Künstlerisches Lehramt und Bildende Kunst in Prozent

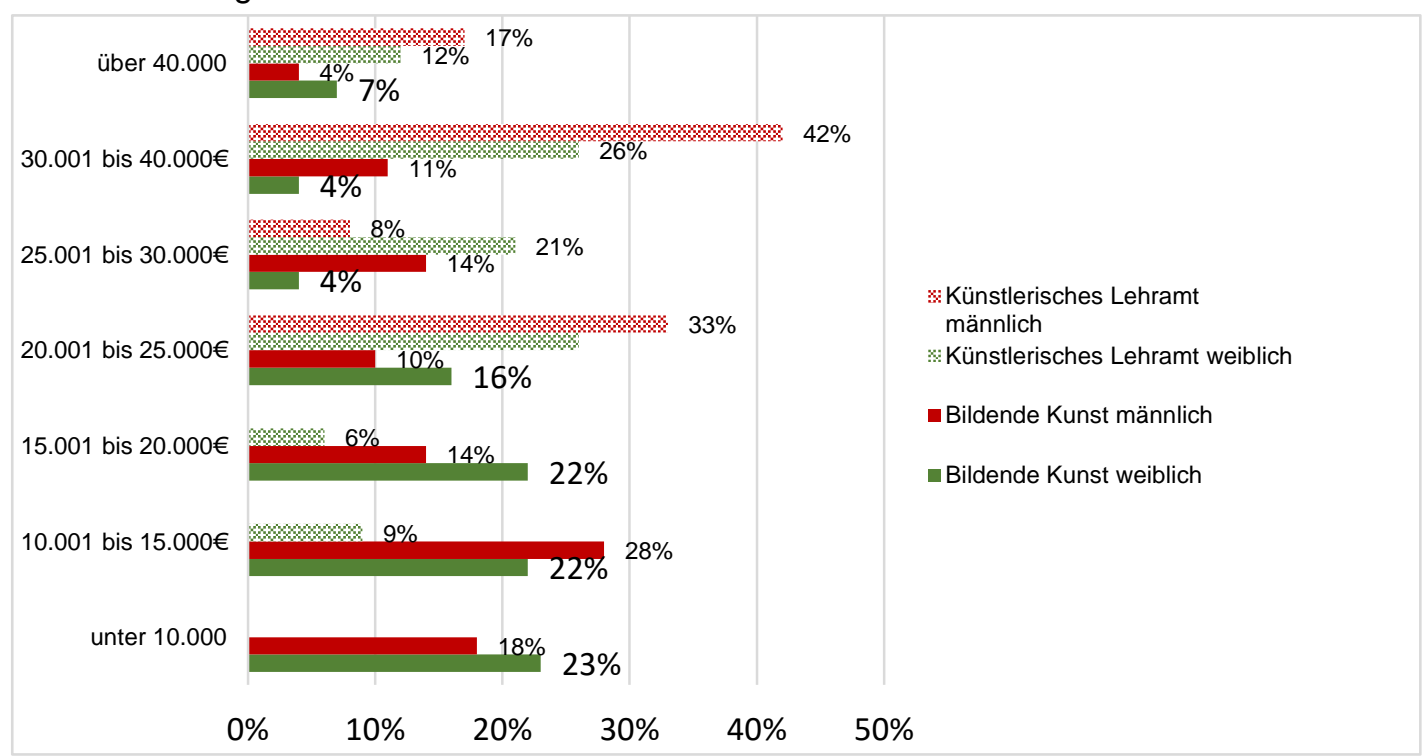

Quelle: ZSI - Absolvent_innenstudie 2000-2018, eigene Berechnungen

Allerdings lassen sich nach den verschiedenen absolvierten Studienrichtungen auch unterschiedliche Streuungen des Einkommens feststellen. Das bedeutet, in einigen Studienrichtungen sind die Einkommen relativ gleich verteilt, in anderen treten ungleiche Verteilungen der Einkommenshöhen auf. Um dies abzugrenzen, wurden die Differenzen des Medianeinkommens und des durchschnittlichen Einkommens nach Studienrichtung abgeglichen ${ }^{11}$. Darauf ergibt sich folgendes Bild:

Tabelle 4

Einkommensstreuung nach Studienrichtung

Einkommensstreuung

geringe Einkommensstreuung

mittlere Einkommensstreuung

breite Einkommensstreuung

\section{Studienrichtung}

$\neg$ Künstlerisches Lehramt

$\neg$ Master in Critical Studies

$\neg$ Bildende Kunst

$\neg \quad$ Konservierung und Restaurierung

$\neg$ Bühnengestaltung

$\neg$ Architektur

Quelle: ZSI - Absolvent_innenstudie 2000-2018, eigene Berechnungen

Der soziale Status der Herkunftsfamilie (Elternhaus) hat einen Einfluss auf die individuelle Einkommenssituation. So streuen die Einkommen von Absolvent_innen, deren Eltern einen hohen Sozialstatus aufweisen, wesentlich stärker, als bei jenen mit niedrigem oder mittlerem Sozialstatus. Das bedeutet, dass in dieser Gruppe unabhängig von dem absolvierten Studium viele Unterschiede in der Einkommenshöhe vorliegen. Es ist anzunehmen, dass zwei gegensätzliche Effekte wirken: zum einen können Phasen des niedrigen Einkommens flexibler überbrückt, andererseits hohe Einkommen lukriert werden. Gleichzeitig

\footnotetext{
${ }^{11}$ Das Medianeinkommen gibt jenen Wert an, der genau in der Mitte aller Einkommen liegt. Das Durchschnittseinkommen ist die Summe aller Einkommen durch die Anzahl der Personen bzw. Angaben. Ist die Differenz zwischen Median und Durchschnitt hoch, ist dies ein Hinweis auf eine breite Streuung.
} 
ist es ein Hinweis dafür, dass Absolvent_innen, deren Eltern keinen hohen Sozialstatus aufweisen, sich in der Tendenz eher an abgesicherten Beschäftigungsverhältnissen mit flachen Einkommenskurven orientieren. Die Unterschiede in der Einkommenshöhe sind in dieser Gruppe geringer.

Die Interviews bestätigen diesen Trend. Sie zeigen, dass tendenziell den interviewten Absolvent_innen, die sich selbst in eine Herkunftsfamilie mit niedrigem bzw. mittlerem sozialen Status einordnen, ein stabiles Einkommen wichtig ist. In ihrer Kindheit hätten sie mit wenig Geld auskommen müssen. Das sei eine Erfahrung, die sie in ihrem Erwachsenenleben nicht reproduzieren möchten.

\subsection{Gestaltung der Erwerbsarbeit und Arbeitszufriedenheit}

Die Erwerbsarbeits-Realität der Absolvent_innen der Akademie ist geprägt von der Kombination unterschiedlicher Beschäftigungsverhältnisse und wechselnden Arbeit- und Auftraggeber_innen. Dies drückt sich unter anderem in der Vielfalt der Beschäftigung aus. Unabhängig vom Abschlussjahrgang sind Kombinationen unterschiedlicher Beschäftigungsarten sowohl direkt nach Abschluss des Studiums als auch im zeitlichen Verlauf weit verbreitet. Rund die Hälfte der Befragten gibt für das Jahr 2018 (als zeitnahes Referenzjahr) an, mehrere Beschäftigungsverhältnisse kombiniert zu haben. Zum selben Anteil kombinierten die Befragten Beschäftigungsarten direkt nach dem Studienabschluss.

Eine Herausforderung, die mit der Einkommenshöhe und den wechselnden Beschäftigungsverhältnissen einhergeht, ist der Erwerb von Anwartschaften in der Sozialversicherung sowie das Auftreten von Versicherungslücken. Rund 35\% im Befragungssample konnten keine Sozialversicherung über Erwerbsarbeit abdecken und sind im Jahr 2018 entweder freiwillig selbstversichert, mitversichert oder haben gar keine Sozialversicherung. 24\% erhielten Zuschüsse aus der Künstler_innen-Sozialversicherung.

Die befragten Absolvent_innen der Akademie gehören zu jenen Menschen mit vergleichsweise hohen Arbeitszeiten. Die durchschnittliche Wochenarbeitszeit in Erwerbsarbeit liegt bei 41,1 Stunden. Auffällig ist, dass auch jene, die angeben Teilzeit beschäftigt zu sein, im Mittel 37,7 Stunden in der Woche erwerbsarbeiten. Zwischen Frauen und Männern unterscheiden sich die Angaben zum Erwerbsarbeitsumfang pro Woche deutlich.

In der Gegenüberstellung der durchschnittlichen Wochenerwerbsarbeitszeiten von Frauen und Männern beträgt die Differenz 4,3 Stunden. Werden jedoch Lebenslagen bzw. Haushaltssituationen in die Betrachtung miteinbezogen, zeigt sich deutlich, dass insbesondere Betreuungspflichten starken Einfluss auf die Wochenstunden in Erwerbsarbeit von Frauen haben. Die Wochenerwerbsarbeitszeit von Frauen mit Betreuungspflichten liegt deutlich unter dem Durchschnitt, jene der Männer darüber. 
Abbildung 5

Durchschnittliche Wochenstunden in Erwerbsarbeit nach Geschlecht (binär) und Haushaltszusammensetzung

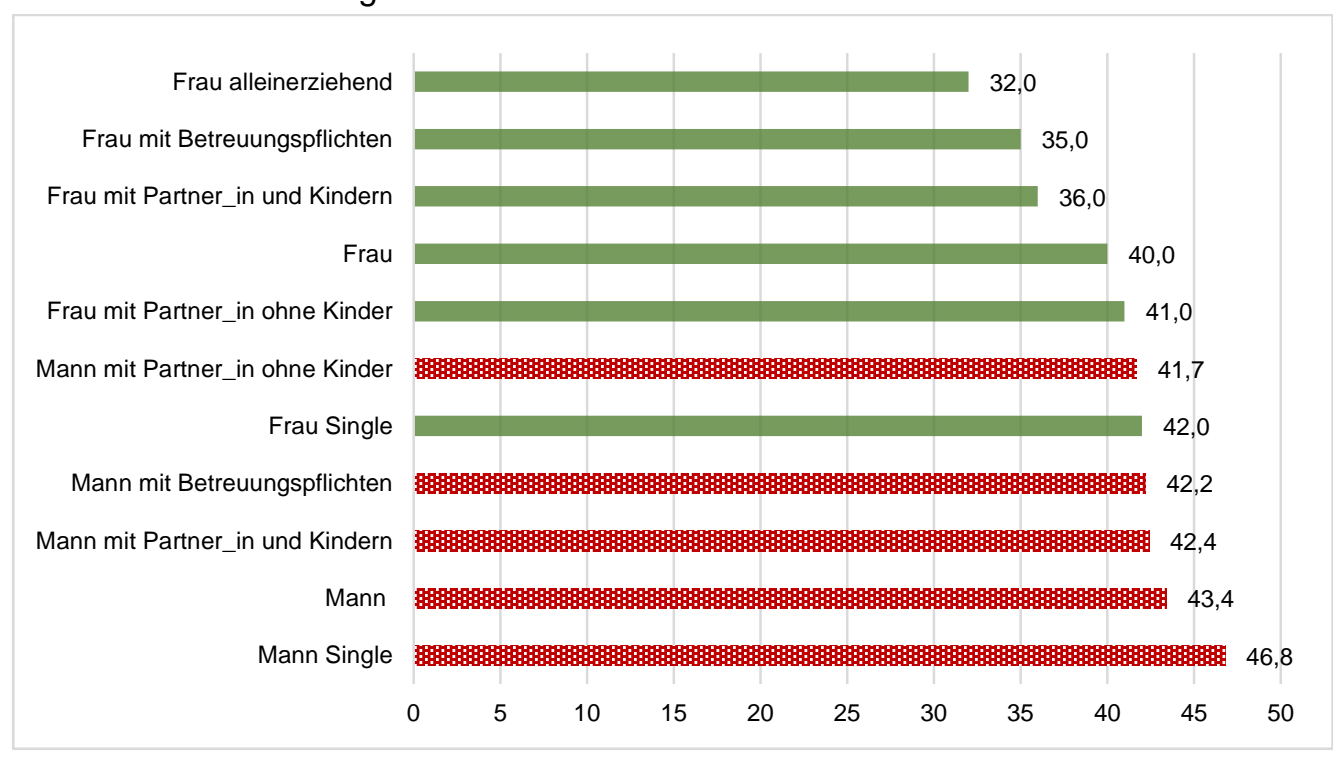

Quelle: ZSI - Absolvent_innenstudie 2000-2018

Auch nach Studienrichtungen unterscheiden sich die durchschnittlichen Wochenerwerbsarbeitszeiten. Dabei liegen die Befragten mit Studienabschlüssen in der Bildenden Kunst, der Architektur und der Bühnengestaltung über dem Durchschnitt, jene der Konservierung und Restaurierung und des Künstlerischen Lehramts darunter. Allerdings ist dabei zu beachten, dass bei der individuellen Einschätzung von Arbeitszeiten auch Fragen der Arbeitsorganisation Einfluss haben. So sind tendenziell in Bereichen mit relativ hoher Institutionalisierung die Arbeitszeiten niedriger bewertet als in sehr projektförmigen oder selbstorganisierten Bereichen.

Die Abgrenzung zwischen beruflicher Tätigkeit und Freizeit ist nicht immer ganz eindeutig für die Befragten. In den Interviews wird dies vor allem im Hinblick auf die Arbeit in Netzwerken und auf einen Anspruch an hohe Präsenz bei Veranstaltungen und Events sowie an eine stetige Selbstvermarktung thematisiert.

Dieses Netzwerk aufbauen, das ist jetzt nicht immer nur Arbeit, sondern kann wirklich sehr oft auch ein Spaß sein. Man geht mal abends was essen oder was trinken. Oder man trifft sich auf einen Kaffee oder ein Frühstück und redet dann halt noch währenddessen über Kampagnen oder Sachen, die man zusammen umsetzen könnte. (Absolventin des Künstlerischen Lehramts, Studienabschluss 2019)

Diese Anforderung an Präsenz und die fließenden Übergänge zwischen Erwerbsarbeit und Freizeit werden in den Interviews kritisch hinterfragt. Einerseits betonen die Interviewten, dass sich Netzwerke eher durch gemeinsame Projekte und Formen des künstlerischen Arbeitens ergeben. Andererseits sehen sich gerade jene Interviewten mit Betreuungspflichten vor allem bei der Teilnahme an Events und der damit verbundenen Netzwerksbildung eingeschränkt. Gleichzeitig empfinden jene ohne Betreuungspflichten die Präsenz bei Events überbewertet und sprechen in diesem Zusammenhang von einem „Mythos“.

Kinderbetreuung ist vor allem für die weiblichen Interviewten ein Thema, dass sie in ihrer Erwerbstätigkeit vor Herausforderungen stellt. Die befragten Frauen thematisierten in Bezug auf ihre Mutterschaft die Notwendigkeit ein stabiles und ausreichendes Einkommen zu 
erzielen. Sie formulieren gleichzeitig ihre Zerrissenheit zwischen den unterschiedlichen Ansprüchen und ihre Angst im Kunstbetrieb nicht mehr reüssieren zu können.

Also, die klare Angst davor, dass man als Künstlerin nicht mehr mithalten kann,- als Mutter - die ist definitiv da, da bin ich mir ganz sicher.

(Absolventin der Bildenden Kunst, Studienabschluss 2016)

Ich habe eine fünfjährige Tochter. Und zum ersten Mal auch gesehen, dass es mit Kind wirklich wahnsinnig schwer ist. Weil ich dann über meine Grenzen gehen muss und teilweise auch über ihre. (Absolventin der Bühnengestaltung, Studienabschluss 2004)

Andere Frauen thematisieren, dass sie nicht in ihrem Tempo studieren oder arbeiten könnten, sondern ihre Arbeitsorganisation den Anforderungen der Elternschaft unterordnen müssen.

Von den interviewten Männern geht nur einer auf die Frage von Familie und Erwerbsarbeit in einem künstlerischen Umfeld ein.

Es ist einfach kein Wunder, dass die Scheidungsrate bei Architekten ziemlich hoch ist. Weil es zwingt dich eigentlich ins klassische Familienbild rein. Meine Frau ist selbständig in einem künstlerischen Beruf. Und sie hat nicht so viele Aufträge, dass sie da ständig beschäftigt ist. Aber das ist halt das klassische Rollenbild und das wollten wir nie haben. Ich bin der, der hauptsächlich verdient, sie muss halt die Familie checken.

(Absolvent der Architektur, Studienabschluss 2001)

Die Frage nach belastenden Faktoren in Zusammenhang mit der Erwerbsarbeitssituation wird insgesamt mittel eingeschätzt. Auf der vorgegebenen Skala von $1=$ geringer Belastung bis $5=$ hoher Belastung bewegen sich die Mittelwerte rund um die Werte 2,0 und 2,5. Damit werden die Faktoren zwar als Belastung empfunden, aber nicht als übermäßig hinderlich eingeschätzt.

Wie die nachstehende Abbildung 6 zeigt, ist für beide Geschlechter die Erwerbsprekarität die stärkste Belastung. Allerdings bewerten Frauen die Belastungsfaktoren höher als Männer. Insbesondere die Erwerbsprekarität und die schwierige Vereinbarkeit von Beruf mit Betreuungspflichten werden von Frauen als stärker belastend empfunden. 
Abbildung 6

Belastungsfaktoren in Bezug auf die Erwerbssituation nach Geschlecht (binär), Mittelwerte der Belastungsstärke

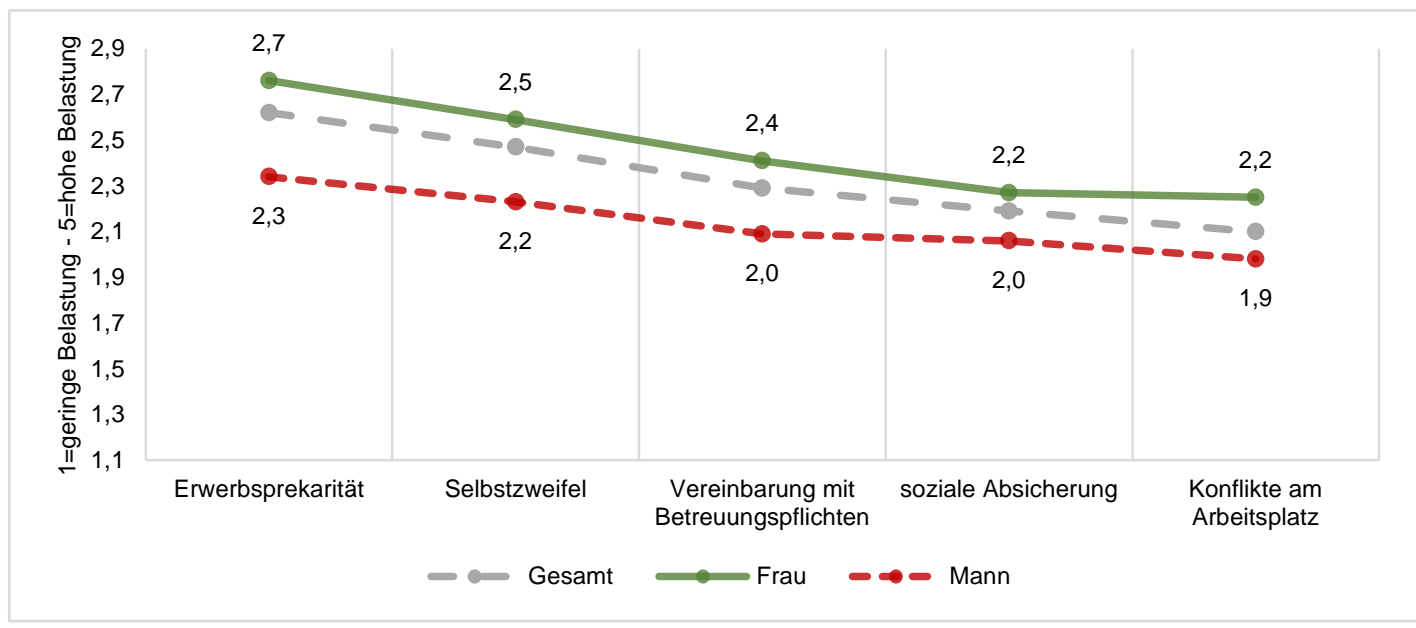

Quelle: ZSI - Absolvent innenstudie 2000-2018

Um die Einschätzung der Arbeitszufriedenheit ${ }^{12}$ abzubilden, wurden verschiedene Faktoren dahingehend abgefragt, ob sie aus Sicht der Absolvent_innen als wichtig angesehen und als erfüllt empfunden werden (Abbildung 7). Für die Auswertung sind diese Items in thematische Gruppen ${ }^{13}$ zusammengefasst.

Die größte Kluft zwischen Wunsch bzw. Anspruch und Wirklichkeit zeigt sich bei der Frage nach dem existenzsichernden Einkommen. 96\% der Befragten finden dies einen wichtigen Faktor in Bezug auf die Arbeitszufriedenheit. Es geben jedoch lediglich $53 \%$ an, dass sie diesen auch als erfüllt ansehen.

Frauen bewerten den Faktor eines existenzsichernden Einkommens weniger erfüllt als Männer. Er wird zwar von beiden Geschlechtern als gleich wichtig eingeschätzt, doch geben $51 \%$ der befragten Frauen und 56\% der befragten Männer an, dass sie ein existenzsicherndes Einkommen erzielen. Befragte, die sich keiner binären Geschlechterkategorie zuordnen, erzielen zu 43\% ein Einkommen, das ihre Existenz sichern kann.

Noch deutlicher wird die Differenz zwischen den Geschlechtern beim Faktor der Vereinbarkeit von Privatem und Beruf (in der nachstehenden Grafik subsumiert unter „Arbeitsumfeld"). Diese Vereinbarkeit sehen 50\% der Frauen und 60\% der Männer als zufriedenstellend erfült an. Sich nicht binär zuordnende Personen bewerten zu 71\% diesen Faktor als wichtig und erfüllt.

12 Die Fragestellungen lauten: Wie wichtig sind Ihnen folgende Faktoren bei Ihrer Arbeit? Und inwieweit sehen Sie diese Faktoren in Ihrer derzeitigen Arbeitssituation erfüllt?

$13 \mathrm{Vgl}$. Tabelle A1 für die Ergebnisse der einzelnen Faktoren. Folgende Indikatoren wurden gruppiert:

$\neg \quad$ Kreativität: Freude an der Arbeit, kreativ sein, abwechslungsreiche Tätigkeiten;

$\neg \quad$ gesellschaftliche Wirksamkeit: gesellschaftlich wirksam zu sein, sinnvolle Arbeit;

$\neg$ Qualifikation/Entwicklung: meine im Studium erworbenen Qualifikationen einsetzen, persönliche Entwicklungsmöglichkeit;

$\neg \quad$ Arbeitsumfeld: gutes Arbeitsklima, Vereinbarkeit von Privatem und Beruf, gute Ausstattung mit Arbeitsmitteln, Teamarbeit, geregelte Arbeitszeiten;

$\neg \quad$ Anerkennung: Anerkennung für meine Arbeit, persönliches Ansehen, hohes Einkommen. 
Abbildung 7

Wichtige Faktoren der Arbeitszufriedenheit und deren Erfüllungsgrad in Prozent

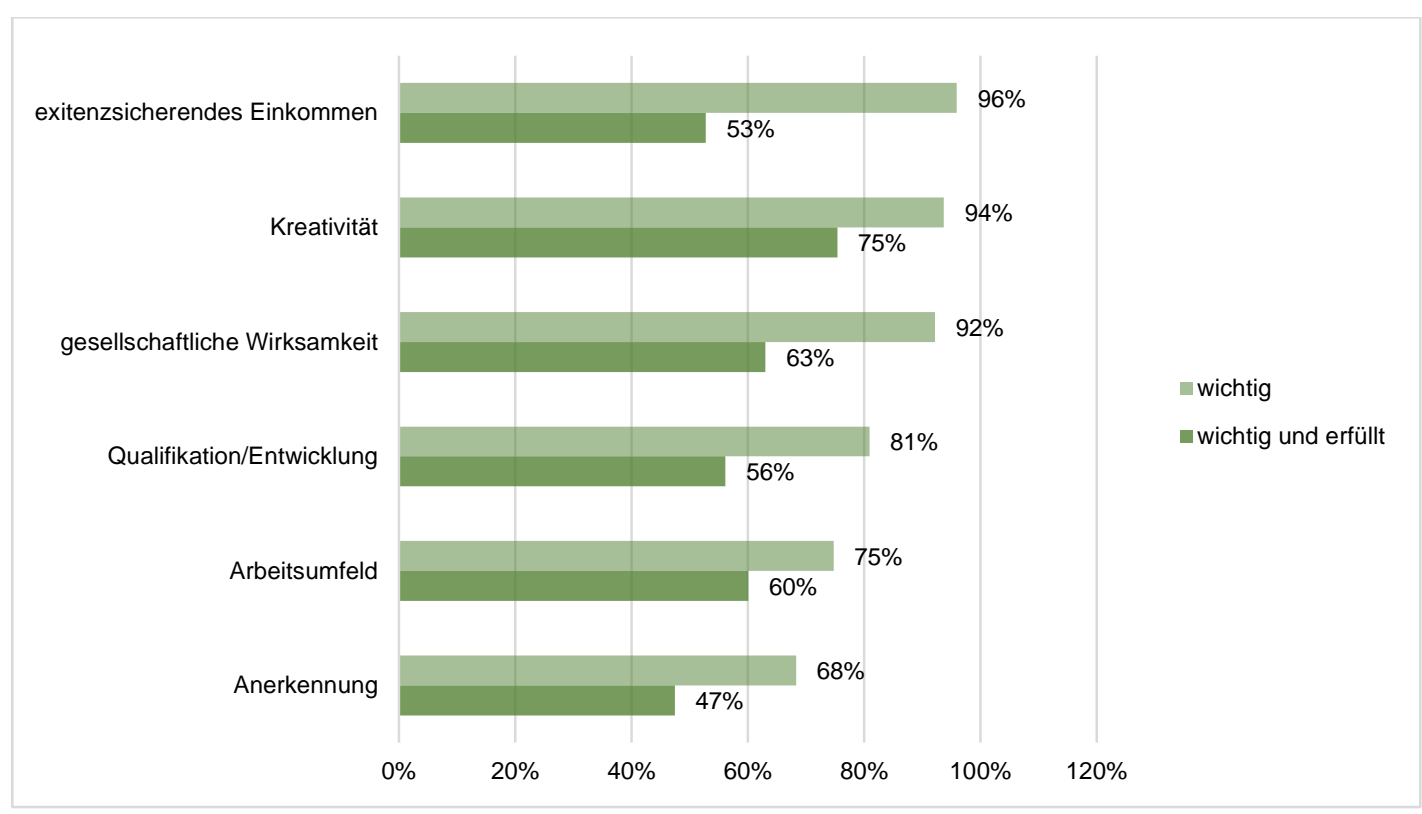

Quelle: ZSI - Absolvent_innenstudie 2000-2018, eigene Berechnungen

\subsection{Zwischenfazit}

Zusammenfassend kann die Erwerbssituation der Absolvent_innen im Jahr 2018 wie folgt beschrieben werden: Es gelingt der Mehrheit in einem studienbezogenen Feld der Erwerbsarbeit Fuß zu fassen. Allerdings sind viele mit prekärer und hybrider Beschäftigung der gleichzeitigen Ausübung von mehreren Beschäftigungsarten - konfrontiert. Die Einkommen streuen zum Teil weit. Bei der Mehrheit der Befragten liegen die mittleren Einkommen deutlich unter dem Medianeinkommen in Österreich.

Die Arbeitsrealität zeichnet sich durch einen hohen Grad an Selbstorganisation sowie wechselnde Beschäftigungsverhältnisse und Vertragsformen aus. Die Erwerbs-Arbeitszeit liegt eher über dem Durchschnitt, jedenfalls aber über der Normalarbeitszeit in Österreich von 38,5 bis 40 Wochenstunden. Belastungen aus der Arbeitsrealität im Kultur- und Kunstbereich werden wahrgenommen und thematisiert, jedoch als weder besonders hoch noch besonders gering eingeschätzt.

Die gesellschaftliche Arbeitsteilung zwischen Frauen und Männern spiegelt sich auch bei den befragten Absolvent_innen wider: Frauen verdienen weniger, haben weniger Freiraum in Bezug auf die Gestaltung von bezahlten und unbezahlten Arbeitszeiten und fühlen sich belasteter.

Die Einschätzung der Arbeitszufriedenheit ist zwischen den Geschlechtern ähnlich. Ausnahmen zeigen sich bei Fragen des existenzsichernden Einkommens und der Vereinbarkeit von Privatem und Beruf. Frauen sehen ein existenzsicherndes Einkommen weniger erfüllt als Männer. Personen, die sich keiner binären Geschlechtskategorie zuordnen, weisen bei diesem Faktor die niedrigsten Werte auf. 


\section{BERUFLICHE WEGE SEIT DEM STUDIENABSCHLUSS}

\section{1 Übergang in die Berufswelt}

Der Übergang vom Studium in die Arbeitswelt erfolgt in der Regel fließend, da ein Großteil bereits während des Studiums erwerbstätig war. Über 70\% der Befragten ist bereits in den ersten sechs Monaten nach dem Studienabschluss in einem studienbezogenen Tätigkeitsfeld beschäftigt. In vielen Fällen wird die Erwerbstätigkeit direkt an das Studium angeschlossen. Zeiten der Abschlussarbeit überschneiden sich mit beginnender Berufstätigkeit im studienbezogenen Feld.

Abbildung 8

Einschätzung des Übergangs in die Berufswelt nach Geschlecht (binär) in Prozent

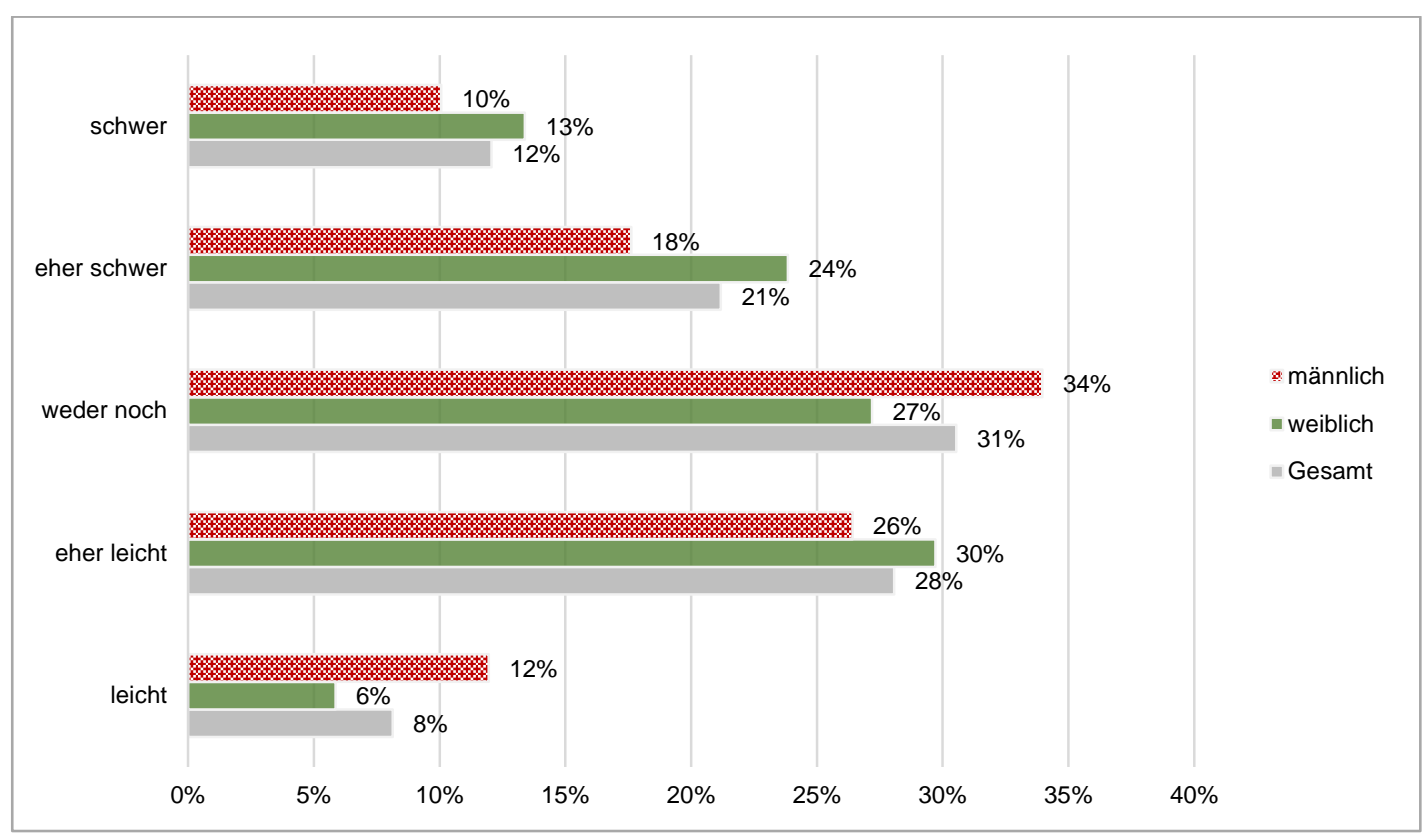

Quelle: ZSI - Absolvent_innenstudie 2000-2018

Die Befragungsergebnisse zeigen: Der Übergang gelingt am leichtesten, wenn während des Studiums eine Erwerbstätigkeit im studienbezogenen Feld ausgeübt wurde. Dennoch wird eine Reihe von Herausforderungen problematisiert. Am häufigsten werden die Bezahlung von Erwerbsarbeit (44\%) und unzureichende Jobangebote (36\%) angegeben, wie Abbildung 9 illustriert. Die Frage der Vereinbarkeit von Privatem und Beruf ist zur Zeit des Studienabschlusses noch kein so entscheidendes Thema. 
Herausforderungen beim Übergang in die Berufswelt (Auswahl) nach Geschlecht (binär) in Prozent

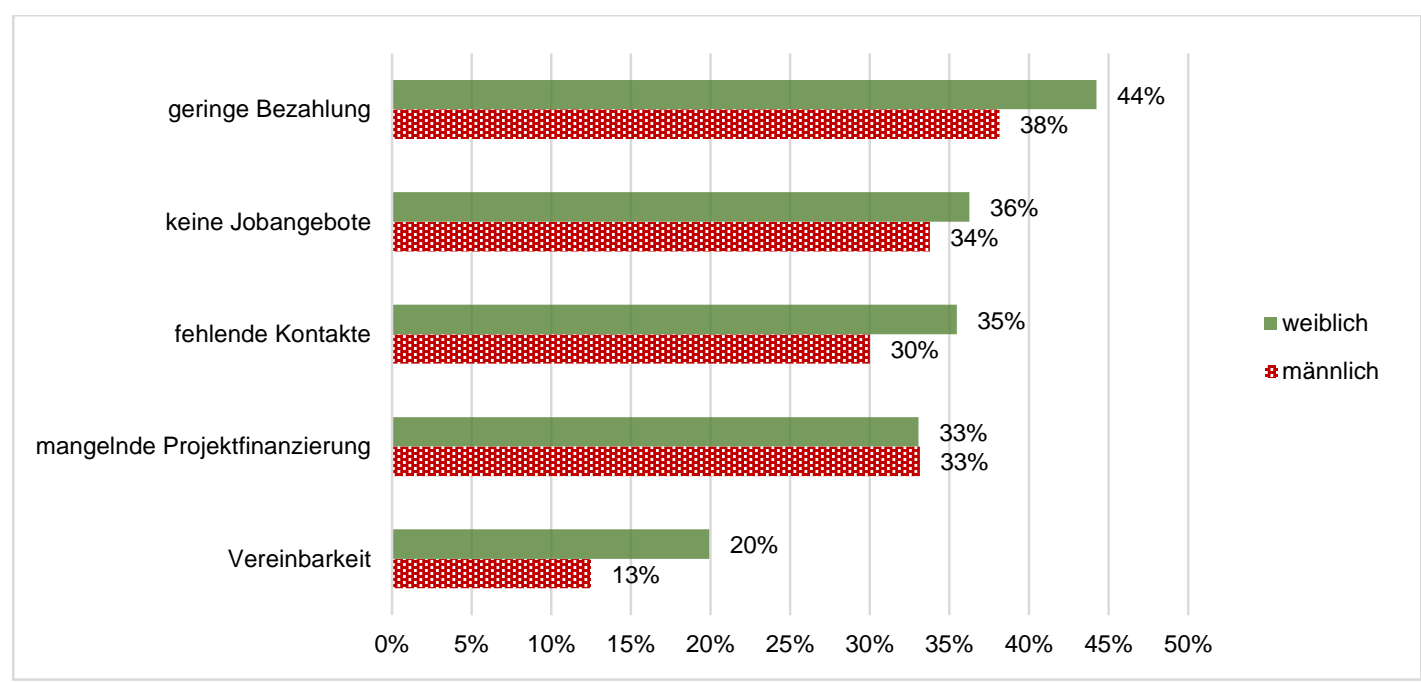

Quelle: ZSI - Absolvent_innenstudie 2000-2018

Längerfristig betrachtet bleiben die befragten Absolvent_innen in einem studienbezogenen Tätigkeitsfeld erwerbstätig. Unabhängig vom Zeitpunkt des Studienabschlusses sehen der überwiegende Teil der Befragten in ihrem Tätigkeitsfeld einen engen oder teilweisen Studienbezug (69\% bzw. 21\%). Dieser Befund ist nach Abschlusskohorten stabil, Absolvent_innen, deren Studien-Ende bis zu 20 Jahre zurückliegt, schätzen den Studienbezug ihrer Erwerbsarbeit sehr ähnlich ein, wie jene, die ihr Studium erst vor kurzem (bis zu zwei Jahre) abgeschlossen haben.

\subsection{Erfolge und Brüche}

Die ersten - von den Befragten selbst als solche definierten - Erfolge in einem studienbezogenen Feld treten rund um den Studienabschluss auf, unabhängig davon, wie lange dieser zurückliegt. Dies ist ein Indiz dafür, dass bereits während des Studiums Aktivitäten im studienbezogenen Berufs- und Tätigkeitsfeld gesetzt werden. Es spricht für eine enge Anbindung der Akademie an das künstlerische Umfeld und an die Praxis.

Rückschläge und Brüche in der Erwerbsbiografie werden, ähnlich wie die Schwierigkeiten in Bezug auf den Berufseinstieg, vor allem in Bezug auf prekäre Beschäftigung, Arbeitslosigkeit und finanzielle Probleme thematisiert. Das weist auf eine dauerhafte Problematik in der Erwerbsbiografie der befragten Absolvent_innen hin. Frauen unterbrechen häufiger als Männer ihre Erwerbstätigkeit aus Gründen der Kinderbetreuung. Sie sind auch häufiger von Arbeitslosigkeit betroffen, ein Umstand, der weitgehend in allen Berufsfeldern, aber insbesondere in einem Berufsfeld mit vergleichsweise hohen und flexiblen Arbeitszeiten - wie der künstlerischen Arbeit - auch auf Betreuungspflichten zurückzuführen ist.

Interessant ist in diesem Zusammenhang, dass Frauen Brüche in der Erwerbsbiografie wesentlich häufiger auf unzureichende Ausbildung zurückführen, wohingegen Männer verstärkt äußere Faktoren, wie z.B. zu wenig Fördermöglichkeiten thematisieren (Abbildung 10). 
Abbildung 10

Gründe für Brüche in der Erwerbsbiografie (Auswahl) nach Geschlecht (binär)

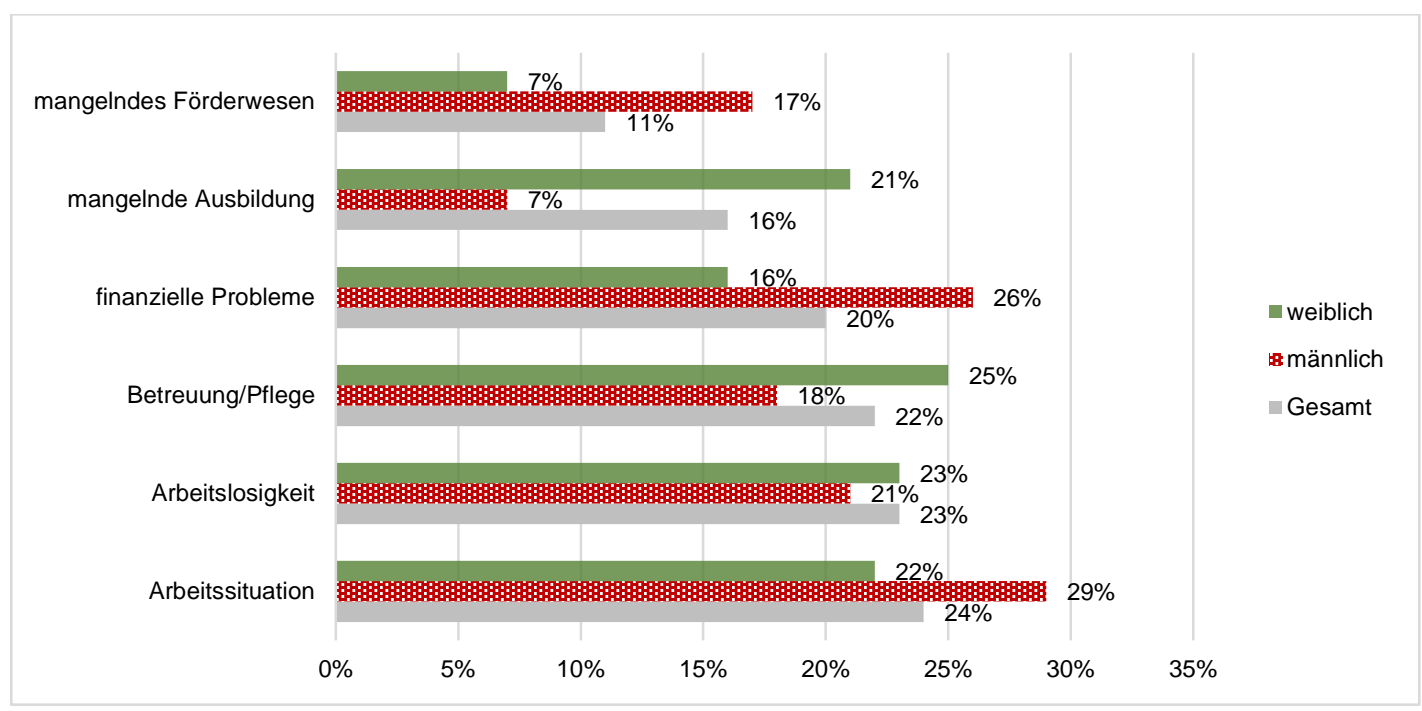

Quelle: ZSI - Absolvent_innenstudie 2000-2018

In den Interviews wird die Familiengründung als wichtiger Einschnitt der Berufslaufbahn immer wieder thematisiert. Die Vereinbarkeit der projektförmigen Arbeit mit Kindern ist herausfordernd. Wiederum sind es in diesem Bereich hauptsächlich die Frauen, die ihre Position reflektieren und Schwierigkeiten thematisieren. Sei es, dass sie für sich nicht ausreichende Zeit für das Studium zu Verfügung hatten, da sie Einkommen lukrieren mussten. Sei es, dass sie ihre persönliche und politische Positionierung durch die Sorgearbeit als beschnitten oder zumindest hinterfragt, aber auch bereichert empfinden.

Ich glaub, das Muttersein hat für mich etwas in mein Leben gebracht, das ich vorher - gerade in diesem ganzen Diskurs, kopfiges Geistzeugs an der Akademie, das ich vorher nicht gehabt habe. Ich habe mir viel feministisches Wissen angeeignet und habe wirklich versucht dementsprechend zu handeln und zu leben, mich zu bilden. So Sachen wie das Stillen, dass es wirklich so pragmatisch ist, oder dass man einfach eben da sein muss, dass man nicht wegkann. Das sind Sachen, also dafür sollte man eigentlich die ganze Ausbildung haben, dass man da gut handelt und auch gesellschaftliche Stereotype aufbrechen kann und damit arbeiten kann.

(Absolventin der Bildenden Kunst, Studienabschluss 2016)

Ich habe in meiner Arbeit sehr viele Lebensläufe von sehr vielen Frauen gesehen und ich sehe einfach: Viele Frauen haben relativ zerstückelte Biografien, und dass viele Frauen generell eher sich den Lebenslauf zusammenstückeln müssen. 
Männliche und sich nicht binär zuordnende Interviewte beziehen erlebte Brüche und Herausforderungen auf andere Faktoren. Sie thematisieren eher das Finden einer eigenen künstlerischen Position und die Arbeitsorganisation als Familienkonstellationen.

Ich glaube eigentlich, genau an dem, was man eigentlich so macht, wenn man künstlerisch tätig ist und Projekte realisiert: das ist irgendwie halt immer wieder auszufechten. Was eigentlich bedeutet, immer wieder neu zu verhandeln, abhängig von gesellschaftlichen Entwicklungen, von Themen, die sich aufdrängen. Also ich würde jetzt nicht sagen, dass mein Profil „Künstler“ ist, empfinde ich eher als eine Verhandlungsbasis, ist eher offen, aber ist gut.

(Absolvent der Bildenden Kunst, Studienabschluss 2016)

Aber ich glaube, ich könnte sicher viel mehr verdienen, wenn ich wollte. Also jetzt so die queeren Themen, zumal so wie wir sie jetzt gestalten. Ich kenne es von Bekannten, wo die Sachen so dezidiert homoerotisch sind und da gibt es zum Beispiel schon eher eine Käuferschicht oder einen Käuferkreis, wo man mehr verdienen kann, als jetzt bei unseren Sachen.

(Absolvent*in der Bildenden Kunst, Studienabschluss 2017)

Diese Zitate unterstreichen die Ergebnisse, die aus dem untersuchten Sample, sowohl in der qualitativen als auch in der quantitativen Erhebung, herauslesbar sind. Der Zugang und die Einflussfaktoren für den Berufseinstieg unterscheiden sich zwischen den Geschlechtern stärker als zwischen den Studienrichtungen.

\subsection{Faktoren beruflicher Integration}

Neben individuellen Voraussetzungen wirken strukturelle Faktoren auf das Gelingen des Übergangs in ein Berufsfeld nach dem Studium ein. In der Zusammenschau der, in den unterschiedlichen Fragen des Fragebogens identifizierten Merkmale in Bezug auf Beruflichkeit ergeben sich eine Reihe unterschiedlicher Einflussflaktoren auf die berufliche Integration.

Mittels statistischer Modellbildung ${ }^{14}$ kann das Zusammenwirken von unterschiedlichen soziodemografischen Indikatoren, der Ausbildungs- und Berufswege sowie der Lebenslage aufgezeigt werden. Es wird identifiziert, welche Faktoren sich günstig oder ungünstig auswirken, also die Wahrscheinlichkeit einer beruflichen Integration erhöhen oder verringern. Darüber hinaus überprüft das Modell, welche Ergebnisse statistisch signifikant sind. Das bedeutet, diese Faktoren können über die Stichprobe hinaus - auf alle Absolvent_innen der Akademie - bezogen werden können. Die Ausgangsfrage für die Modellbildung ist, welche Faktoren die Integration in ein studienbezogenes Berufs- und Tätigkeitsfeld begünstigen.

Wie die Ergebnisse zeigen, ist die kontinuierliche künstlerische Arbeit ein entscheidender Faktor. Befragte, die angeben, dauerhaft und durchgehend künstlerisch tätig zu sein, sind auch im künstlerischen Umfeld beruflich integriert.

\footnotetext{
${ }^{14}$ Es wurde mittels multivariater Datenanalyse ein Modell der linearen Regression gebildet. In statistischen Rechenprozessen werden jene, ins Modell integrierte Einflussfaktoren identifiziert, die mit einer hohen Wahrscheinlichkeit (95\%) auf die Grundgesamt aller Absolvent_innen der Akademie in der Zeitspanne von 2000 bis 2018 wirken.
} 
Das Geschlecht (binär) hat ebenfalls signifikante Auswirkungen auf die berufliche Integration. Frauen allgemein und insbesondere Frauen mit Betreuungspflichten haben ungünstigere Bedingungen für ihre studienbezogene Arbeit. Wohingegen sich Betreuungspflichten bei Männern günstig auf ihre Integration in dieses Berufs- und Tätigkeitsfeld darstellen. Die Strukturen der bestehenden gesellschaftlichen Arbeitsteilung wirken unverkennbar auch im künstlerischen Umfeld. Die ungünstigen Rahmenbedingungen in Hinblick auf Sorgearbeit fallen auf Frauen zurück. Aber auch ohne Betreuungspflichten sind Frauen benachteiligt. Dies kann auf die allgemeine Diskriminierung von Frauen am österreichischen Arbeitsmarkt bzw. speziell am kunstspezifischen Arbeitsmarkt zurückgeführt werden.

Die Herkunft der Absolvent_innen ist nur in Zusammenhang mit einem niedrigen sozialen Status ihrer Eltern ein signifikanter Faktor für die berufliche Integration. Dann wirkt sich eine Herkunft aus einem Nicht-EU-Staat ungünstig aus. Unabhängig vom sozialen Status ist dies nicht so. Eine Erklärung dafür ist, dass Absolvent_innen mit einem niedrigen sozialen Herkunftsstatus generell eher in stabile, unselbständig Beschäftigung streben. Dabei wirken die restriktiven gesetzlichen Beschränkungen beim österreichischen Arbeitsmarktzugang für Personen aus Nicht-EU-Staaten. Über alle Absolvent_innen gesehen (unabhängig vom sozialen Herkunftsstatus) wird diese Auswirkung unter anderem durch den vergleichsweise hohen Anteil an selbständiger Erwerbstätigkeit, die diesen Beschränkungen nicht unterliegt, entschärft.

Ein weiterer Faktor ist die Studienrichtung. Nach einem Studium des Künstlerischen Lehramts, der Architektur sowie Konservierung und Restaurierung fällt der Einstieg in den studienbezogenen Arbeitsmarkt bzw. in die studienbezogene Erwerbsarbeit leichter. In der Bildenden Kunst stellt sich eine Integration in Berufstätigkeit herausfordernder dar. Dies ist vor allem durch die spezifischen Berufsfelder und deren Rahmenbedingungen begründet. Lehramtsstudien sind eng an die Unterrichtstätigkeit in der Schule gebunden, der Einstieg in den Lehrberuf ist ein explizites Bildungsziel. Im Bereich der Architektur wird eine berufsspezifische Qualifikation erworben, ähnlich organisiert ist das Feld der Konservierung und Restaurierung. Alle drei Studienrichtungen bilden formale Voraussetzungen für diese Berufsfelder ab, ohne die eine Beschäftigung dort kaum möglich ist.

Abbildung 11 umfasst wieder alle Studienrichtungen. Sie zeigt, wie die Teilnehmer_innen der Erhebung die Einflussstärke der abgefragten Faktoren bewerten. Durchhaltevermögen wird als wichtigster Faktor für den Einstieg und den Verbleib in einem studienbezogenen Feld eingeschätzt ${ }^{15}$. Die unter dem Faktor Ausbildung zusammengefassten Antwortmöglichkeiten zielen auf formale Kategorien ab und beziehen sich auf Studien-Abschlüsse bzw. die Auswirkungen der Akademie und ihr Ansehen in Hinblick auf die berufliche Integration. Die dahinterstehende Frage ist, ob ein Studium an der Akademie oder bei einer bestimmten Lehrperson von den Befragten als door opener und Erleichterung für die berufliche Integration angesehen wird. Generell ist davon auszugehen, dass alle hier abgefragten Faktoren durch die Zeit an der Akademie beeinflusst wurden.

\footnotetext{
${ }^{15}$ Vgl. Tabelle A2 für die Ausprägung der einzelnen Faktoren. Folgende abgefragten Faktoren wurden thematisch gruppiert:

$\neg \quad$ persönliche Skills: persönliches Auftreten, sich selbst gut verkaufen können, soziale Kompetenzen;

$\neg \quad$ Wettbewerbe: Beteiligung an Wettbewerben und Ausschreibung, Preise und Stipendien;

$\neg \quad$ Ausbildung: Abschluss einer Kunsthochschule/Kunstuniversität, Ansehen der Akademie, Professor_in bei der_dem ich studiert habe;

$\neg \quad$ Kontakte/Selbstvermarktung: aktives Marketing/Öffentlichkeitsarbeit, Internetplattformen aktiv nutzen, Teilnahme/Beteiligung an formellen Netzwerken, Kontakte zu privaten Sammler_innen und Sponsor_innen, Kontakte zu Vermittler_innen und Veranstalter_innen, Pflege informeller Netzwerke.
} 
Abbildung 11

Einflussfaktoren der Integration in Erwerbstätigkeit in einem studienbezogenen Feld nach eingeschätzter Einflussstärke, Mittelwerte

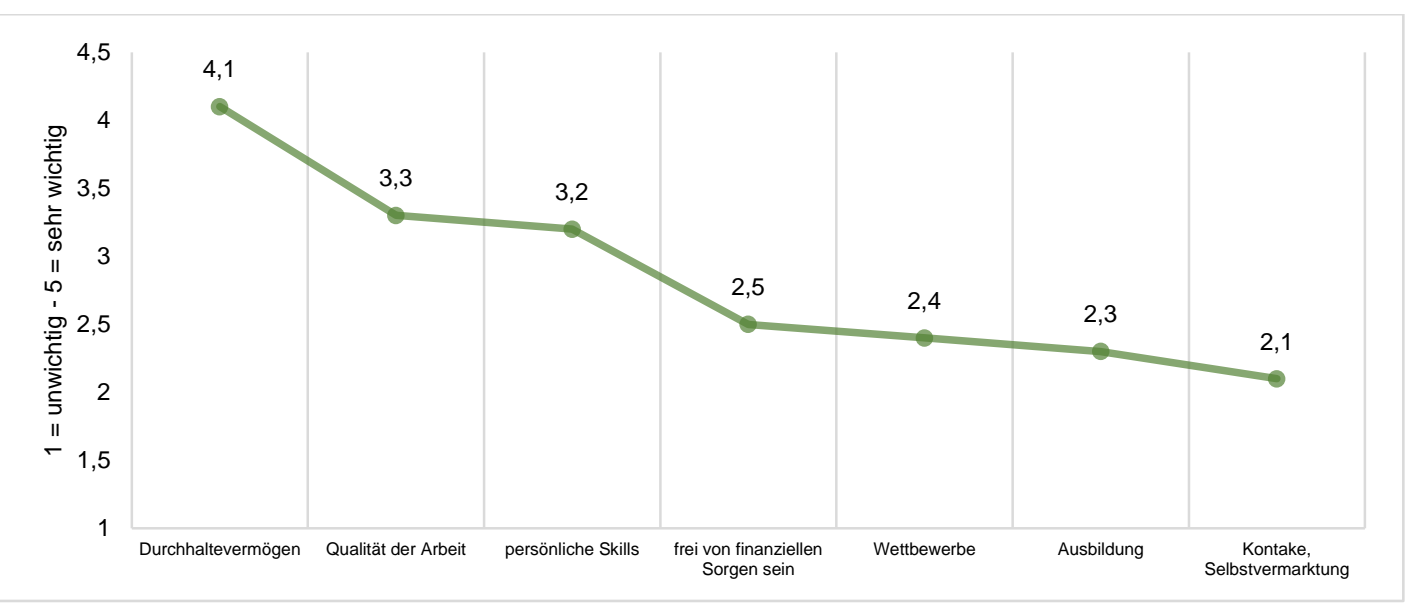

Quelle: ZSI - Absolvent_innenstudie 2000-2018, eigene Berechnungen

Die Ergebnisse zeigen, dass die befragten Absolvent_innen der Qualität der Arbeit und persönlichen Skills (neben dem bereits erwähntem Durchhaltevermögen) einen hohen Stellenwert für die berufliche Integration beimessen. Damit werden individuelle Kompetenzen höher bewertet als etwa die Anerkennung durch Wettbewerbe. Das weist darauf hin, wie wichtig die Entwicklung einer eigenen künstlerischen, beruflichen Position gesehen wird. Kompetenzen, die eine Einschätzung der eigenen künstlerischen Arbeit und deren (Markt)Wert ermöglichen und Selbstvertrauen sind aus Sicht der Befragten wichtig für diese Positionierung. Welchen Einfluss die Studienzeit und die Akademie auf das Herausbilden dieser Kompetenzen hat, wird in Kapitel 5 vertieft dargelegt, in dem die befragten Absolvent_innen ihre Erfahrungen während des Studiums reflektieren.

\subsection{Berufliche Belastungsfaktoren}

In der Erhebung wurden die Teilnehmer_innen der Befragung gebeten, verschiedene Belastungsfaktoren danach einzuschätzen, wie stark diese Auswirkungen auf ihren beruflichen Werdegang hatten. Diese Fragestellung zeigt auf, wie die befragten Absovent_innen einzelne Schwierigkeiten im künstlerischen Umfeld empfinden. Daraus können Rückschlüsse auf Unterstützungsbedarf gezogen werden. Für die Darstellung in Abbildung 12 wurden die beiden Studienrichtungen Künstlerisches Lehramt und Bildende Kunst ausgewählt. Zum einen ermöglichen die vergleichsweise hohen Fallzahlen eine differenzierte Auswertung. Zum anderen unterschieden sich die Erhebungsergebnisse für Absolvent_innen der beiden Studienrichtungen in Hinblick auf die Bewertung der Erwerbssituation (vgl. Kapitel 3). Das legt die Annahme von unterschiedlichen Einschätzungen der beruflichen Belastungsfaktoren nahe.

Die Ergebnisse dieser Auswertungen legen dar, dass befragte Absolvent_innen der Bildenden Kunst alle abgefragten Faktoren höher einschätzen. Daraus kann der Schluss gezogen werden, dass sie in Hinblick auf ihre berufliche Integration stärker empfundenen Hürden gegenüberstehen als befragte Absolvent_innen des Künstlerischen Lehramts. 
Abbildung 12

Belastungsfaktoren nach ausgewählten Studienrichtungen (Mittelwerte)

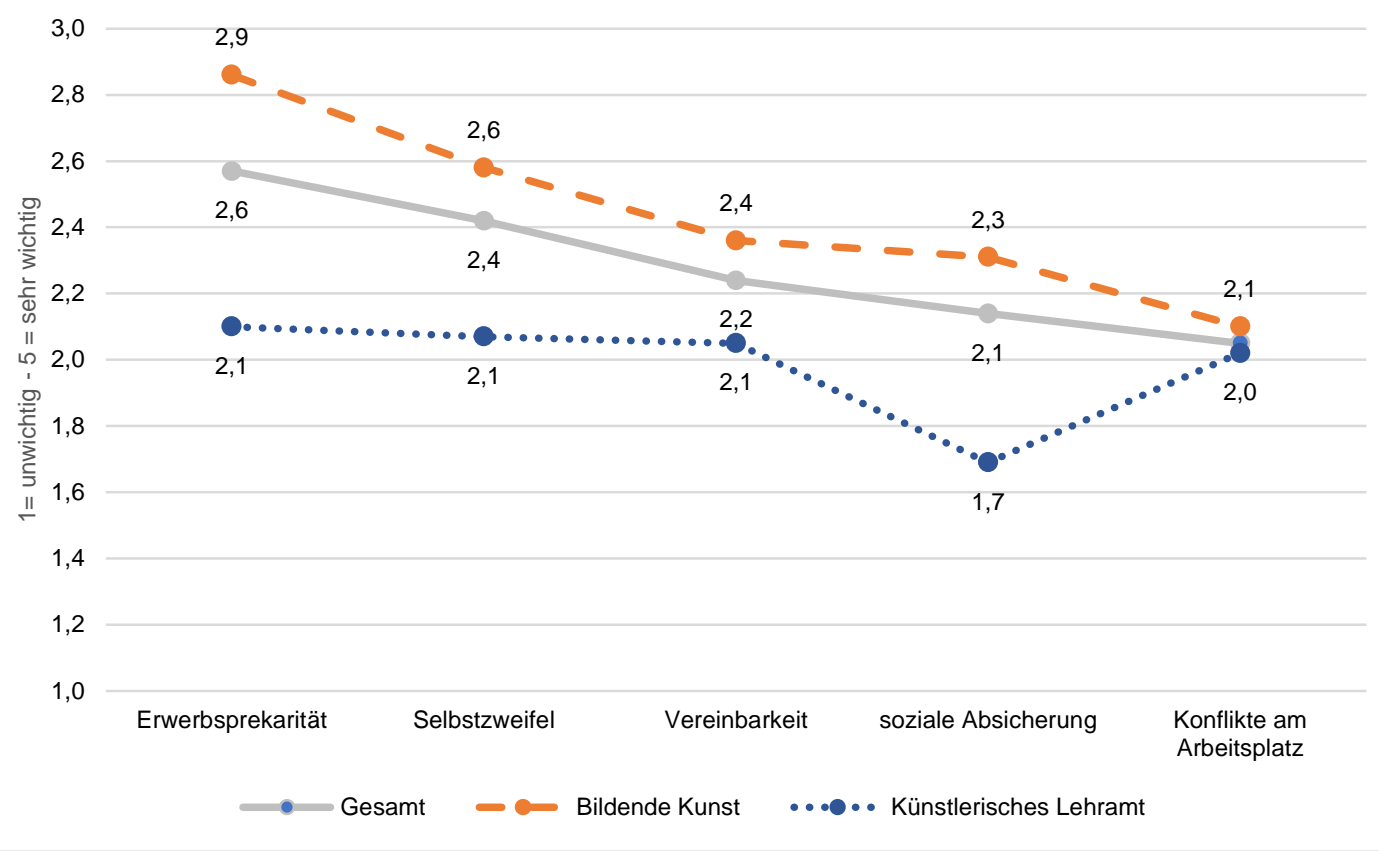

Quelle: ZSI - Absolvent_innenstudie 2000-2018, eigene Berechnungen

Zu den stärksten wahrgenommenen Belastungen gehört die Erwerbsprekarität, gefolgt von Selbstzweifeln. Befragte der Studienrichtung des Künstlerischen Lehramts schätzen die Erwerbsprekarität allerdings als weniger belastend ein. Dies kann in ihrer studienbezogenen Berufstätigkeit in einer Schule oder im Bildungsbereich liegen. Beide Bereiche sind für das Künstlerische Lehramt zwar keineswegs frei von Prekarität, die Beschäftigung ist jedoch häufig vertraglich geregelt, wenn auch nicht unbedingt dauerhaft. Durch diesen Umstand erscheint die Beschäftigungssituation etwas weniger belastend als in dem vielfach wenig reglementierten Bereich der Bildenden Kunst.

Für Absolvent innen der Bildenden Kunst sind Selbstzweifel herausfordernd. Das künstlerische Umfeld verfügt über wenig formale Anerkennungsrituale und -merkmale. Das Feld, in dem es zu reüssieren gilt, ist in seinen Bewertungsvorgaben wenig strukturiert, wenn auch nicht beliebig. Dazu kommt, dass projektförmiges Erwerbsarbeiten, viele unterschiedliche Arbeitsverträge und -formen damit verbunden sind. Absolvent_innen müssen sich häufig wiederkehrend in Bewerbungssituationen und Konkurrenzen begeben.

Auch Absolvent_innen von anderen - an der Akademie angebotenen - Studienrichtungen empfinden Erwerbsprekarität als belastend. Dabei wird deutlich, dass nicht nur niedrige Einkommen, sondern auch geringe Reglementierung in Bezug auf Beruflichkeit ${ }^{16}$ und Vertragsgestaltung Einfluss auf prekäre Lebensumstände haben.

\footnotetext{
${ }^{16}$ Beruflichkeit beschreibt den Zusammenhang zwischen einer formalen Ausbildung und einem damit verbundenen reglementierten Beruf. Im künstlerischen Umfeld ist der Zugang zu einem Beruf nicht so stark an eine formale Ausbildung gebunden, wie in anderen Bereichen. Ausnahmen bilden das Künstlerische Lehramt und die Architektur, für die ein einschlägiges Studium eine Voraussetzung zur Berufsausübung in der Schule bzw. als Architekt_in darstellen.
} 
Und es gab schlimme Zeiten, also es gab finanziell komplizierte Zeiten, Resignation, es gab alles, aber trotzdem habe ich immer noch der, der Kunst, die ich machen wollte, vertraut. (Absolvent der Bühnengestaltung, Studienabschluss 2015)

Also, das ist jetzt auch grad eine Zeit, wo genau diese festen Formen oder dieser feste Beruf, den man immer dachte, man hat ihn am Theater und der ist auch ganz unumstritten - das wird komplett aufgeweicht. (Absolventin der Bühnengestaltung, Studienabschluss 2004)

Diskriminierung als Benachteiligung aufgrund von Zuschreibungen und äußerlichen Merkmalen besteht auch im künstlerischen Umfeld. Die Betroffenheit ist je nach Diskriminierungsgrund ${ }^{17}$ unterschiedlich. Insgesamt lässt sich jedoch festhalten, dass über alle Gründe hinweg, weibliche Absolvent_innen und jene Befragten, die sich keinem binären Geschlecht zuordnen, Diskriminierung stärker thematisieren. Die Auswertungen zu diesen Fragen (vgl. Abbildung 13) legen nahe, dass intersektionale Diskriminierung, also das Zusammenwirken mehrere Diskriminierungsfaktoren, auf die Betroffenen einwirkt.

Abbildung: 13

Betroffenheit von Diskriminierung nach Geschlecht in Prozent

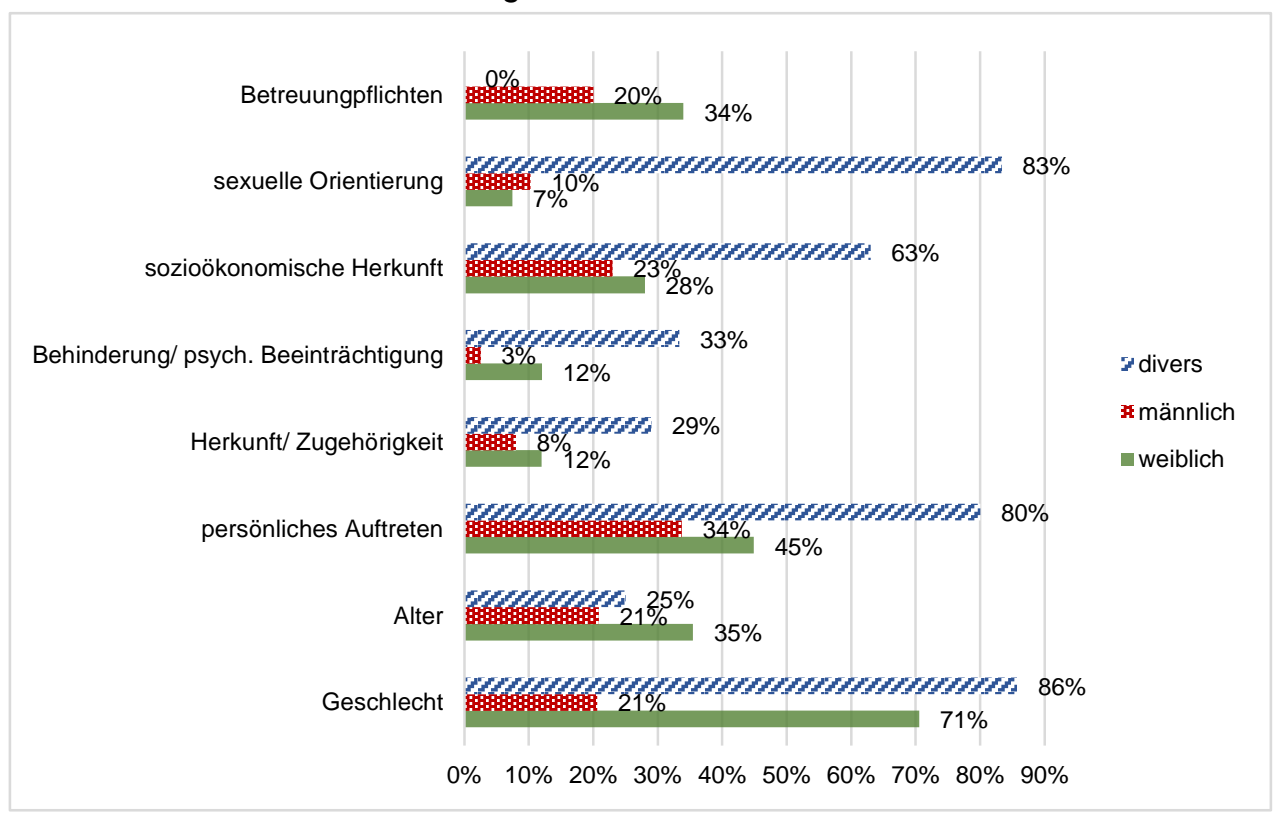

Quelle: ZSI - Absolvent_innenstudie 2000-2018, eigene Berechnungen

Abbildung 14 zeigt die Ausprägung der individuellen Diskriminierung. Diese wurde über die Frage nach der Häufigkeit von Diskriminierungserfahrungen erfasst. Auch wenn die Anzahl der Personen im Sample relativ gering ist, kann tendenziell davon ausgegangen werden, dass Personen, die sich keinem binären Geschlecht zuordnen, häufiger von unterschiedlicher Diskriminierung (intersektional) betroffen sind als z.B. männliche Befragte im Sample. Weibliche Absolvent_innen sehen sich, neben der Diskriminierung aufgrund des Geschlechts, verstärkt Benachteiligungen auf Grund ihrer familiären Situation bzw. von Betreuungspflichten sowie in Bezug auf ihre sozioökonomische Herkunft ausgesetzt.

\footnotetext{
${ }^{17} \mathrm{Vgl}$. Tabelle A3 und A4 für die Ausprägung der einzelnen Diskriminierungsgründe. Folgende Faktoren der Diskriminierungsbelastungen wurden zusammengefasst:

$\neg \quad$ Alter: zu jung, zu alt;

$\neg \quad$ Herkunft/Zugehörigkeit: Sprache, ethnische Herkunft, kulturelle/religiöse Zugehörigkeit, der Ort an dem ich geboren bin.
} 
Abbildung 14

Häufigkeit von Diskriminierungserfahrungen Betroffener nach Geschlecht, Mittelwerte

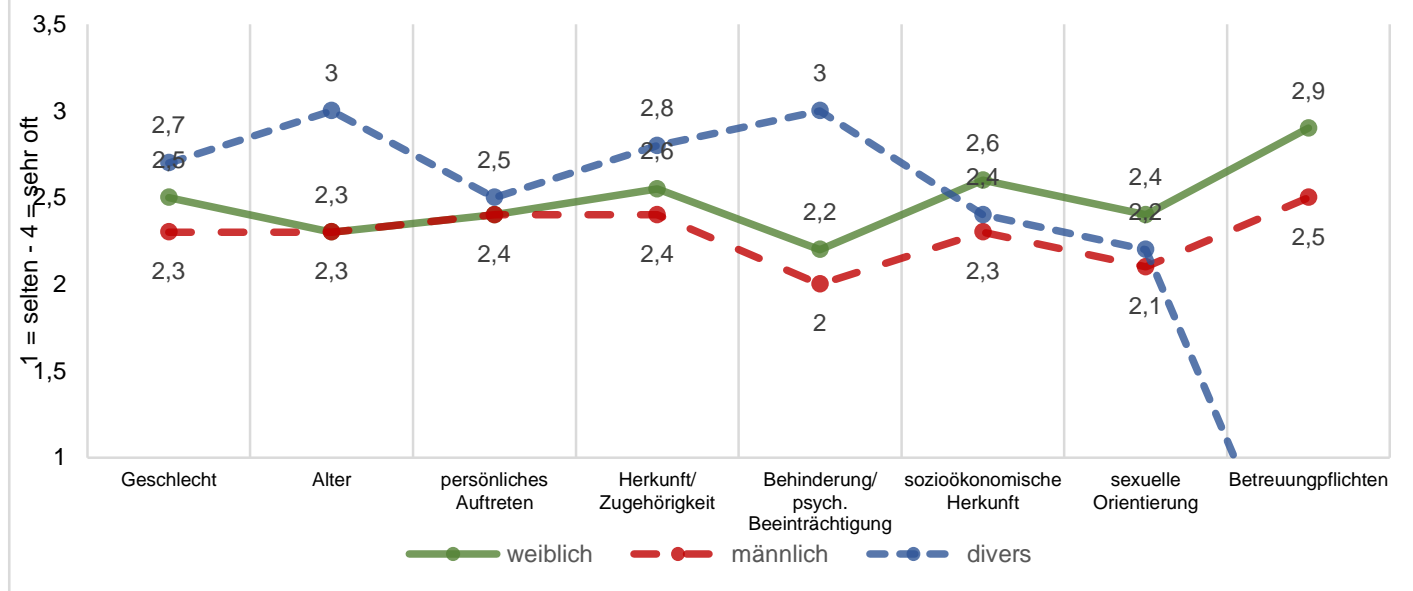

Quelle: ZSI - Absolvent_innenstudie 2000-2018, eigene Berechnungen

In der Darstellung der Diskriminierungsbetroffenheit nach Geburtsort (vgl. Abbildung 15) zeigt die Ausdifferenzierung in der Kategorie „Herkunft/Zugehörigkeit“" ein auffälliges, aber erwartbares Ergebnis. Personen aus Nicht-EU-Staaten sind zu einem vergleichsweise höherem Anteil von Benachteiligungen aus Gründen der Herkunft betroffen.

Abbildung 15

Betroffenheit von Diskriminierung nach Geburtsort in Prozent

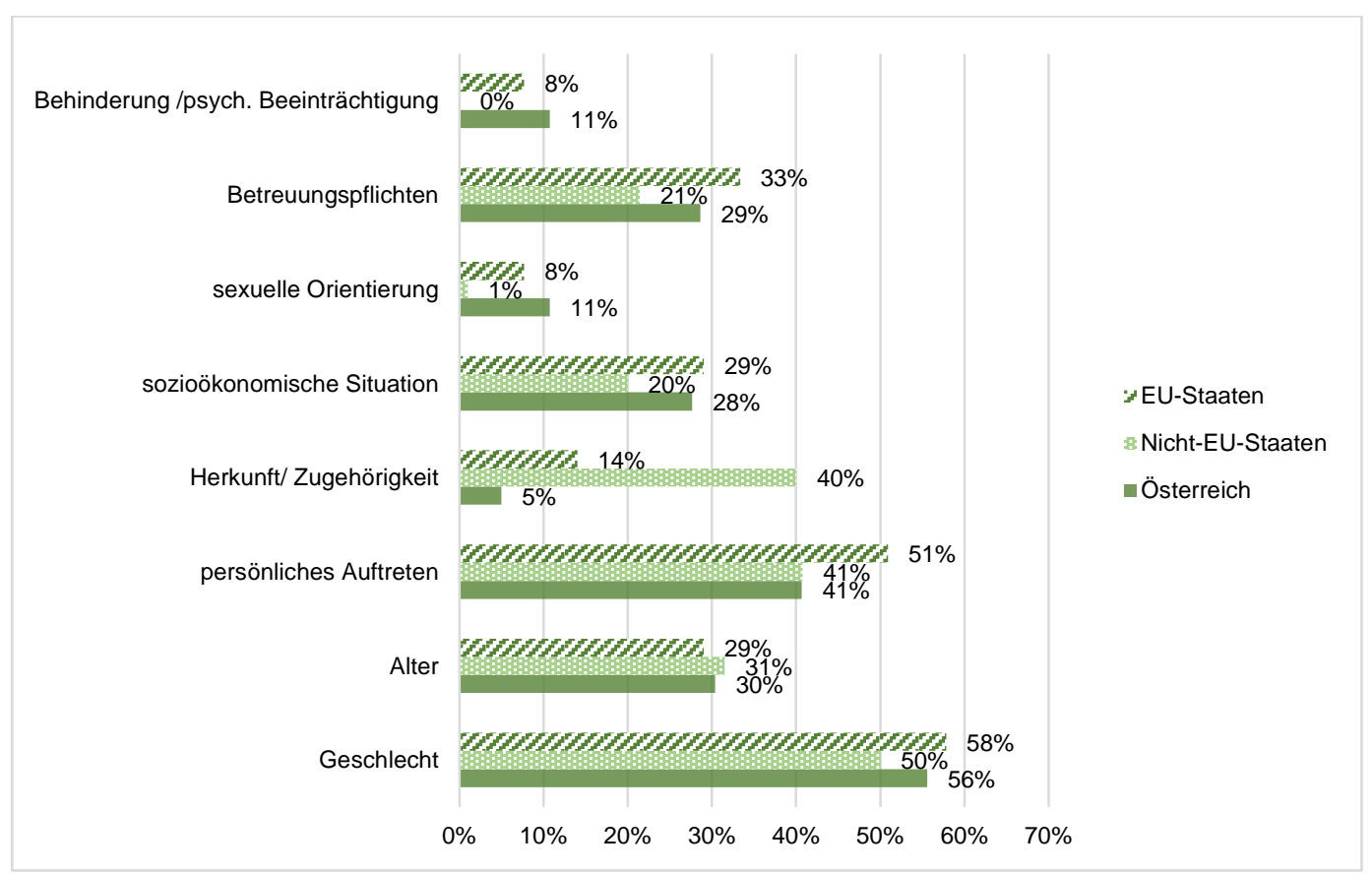

Quelle: ZSI - Absolvent_innenstudie 2000-2018, eigene Berechnungen

Gleichzeitig zeigt sich, dass die Häufigkeit von Diskriminierungserfahrungen aus unterschiedlichen Diskriminierungsgründen bei Befragten, die in Nicht-EU-Staaten geboren sind, wesentlich höher liegt als bei den Vergleichsgruppen. Auch das ist ein Hinweis darauf, dass Diskriminierung oft aus dem Zusammenwirken mehrere Faktoren/Gründe besteht, also intersektional ist. Unabhängig von der Herkunft ergeben sich beim Diskriminierungsgrund in 
Bezug auf das Alter ähnliche Einschätzungen der Betroffenen. Im Sample waren keine Personen vertreten, die angaben, dass ihr Geburtsort in einem Nicht-EU-Staat liegt und die gleichzeitig auf Grund einer Behinderung oder psychischen Beeinträchtigung Diskriminierungen erfahren haben.

Abbildung 16

Häufigkeit von Diskriminierungserfahrungen Betroffener nach Geburtsort, Mittelwerte

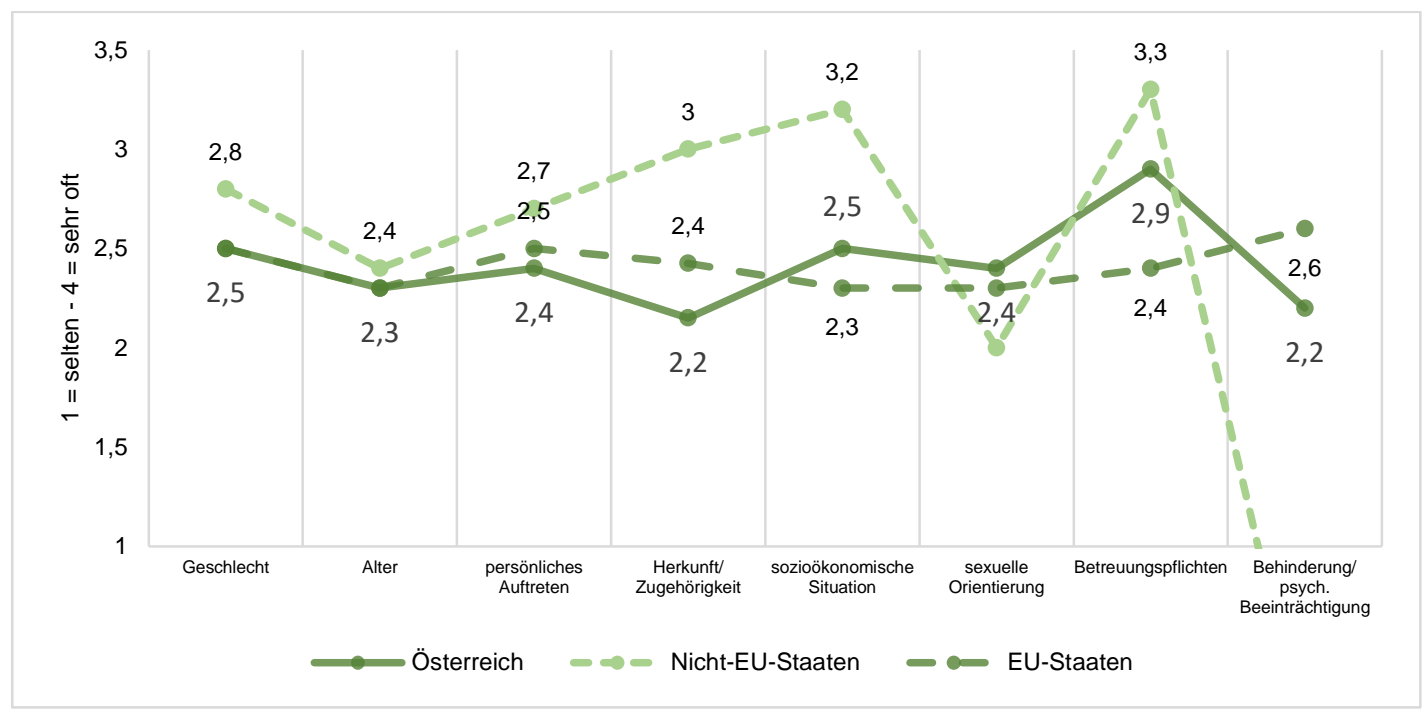

Quelle: ZSI - Absolvent_innenstudie 2000-2018, eigene Berechnungen

In den Interviews tritt hervor, dass eine Form der Diskriminierung in der „Schubladisierung“ oder Exotisierung der eigenen Kunst gesehen wird. Äußere Merkmale oder Zuschreibungen bezogen auf die_den Künstler_in werden mit bestimmten Begriffen und Kunstrichtungen verbunden, auf welche die Betroffenen dann festgelegt werden.

Zum Beispiel gibt es ein paar Projekte afrikanische Projekte - so für mich das ist ein bisschen eine Ghettosituation.

(Absolvent der Bildenden Kunst, Studienabschluss 2010)

[...] dass wir eben jetzt quasi gebucht werden dafür. So: Ahja, das ist ein queeres Künstlerduo. Aber dass wir den Leuten nicht in den Sinn kommen, wenn es jetzt - was weiß ich - um Gruppenportraits geht oder um kunstgeschichtliche Zitate in der Fotografie, wie auch immer.

(Absolvent*in der Bildenden Kunst, Studienabschluss 2017)

Auch der Umgang an der Akademie wurde von den befragten Absolvent_innen nicht frei von Diskriminierung erlebt, obwohl - wie die Interviewten hervorstreichen - die Einrichtung und ihre Beschäftigten in Fragen der Gleichbehandlung einen starken Schwerpunkt setzen. Zu einem gewissen Grad, so legen die Ergebnisse der Interviews nahe, würde es in einem derart engen Betreuungsverhältnis wie an der Akademie und der formalen Bewertungsnotwendigkeit individualisierter und kreativer Arbeit nicht ausbleiben, dass Menschen sich benachteiligt und diskriminiert fühlen. Umso wichtiger und positiver sind die strukturellen Maßnahmen der Akademie zur Unterstützung bei und Verhinderung von Ungleichbehandlung und Benachteiligung zu sehen. 
Weil, vor allem in meiner Diplomarbeit ist mir vorgekommen, auch mit meinem Diplombetreuer, ja, der war einfach echt ein Sexist. Also, der hat mir mehrere Male so irgendetwas gesagt, - ja du präsentierst du irgendwie so ein süßes kleines Mädchen, aber glaub nicht, dass dich des irgendwie retten wird.

(Absolventin, Studienabschluss 2016)

In der Mehrzahl der Interviews wird die Akademie jedoch als sehr sensibel in Fragen von Diskriminierung beschrieben.

Ja, damals hatte ich eigentlich ein bisschen was zu verarbeiten. Na, das war an der Akademie irgendwie eine heile Welt, sozusagen. Anders als was rundherum passiert ist, eben wo ich beschimpft worden bin, oder einmal zusammengeschlagen.

(Absolventin der Bildenden Kunst, Studienabschluss 2011)

Also, die Akademie ist ja wahnsinnig sensibel. Und ist auch der beste Ort, um das Ansprechen von Diskriminierung zu ermöglichen.

(Absolventin der Architektur, Studienabschluss 2016)

In diesem Zusammenhang darf nicht übersehen werden, dass die soziale Herkunft aus Sicht der Befragten starke Auswirkungen aufweist und das künstlerische Umfeld als nicht besonders sozial durchlässig angesehen wird. Dabei gehe es vornehmlich um das Ineinandergreifen von ökonomischem (z.B. Einkommen), kulturellem (z.B. Bildung) und sozialem (z.B. Netzwerke) Kapital. Je besser die Ausstattung damit, desto leichter entwickle sich ein Selbstbewusstsein und desto eher gelinge der Einstieg ins künstlerische Umfeld, dass von einem hohen Grad an symbolischem Kapital durchdrungen sei.

Dann merkt man halt, dass andere Menschen schon damit aufgewachsen, dass sie in Jugendtheater gehen. Man hat schon oft gemerkt, dass es Unterschiede gibt, vielleicht auch im Selbstbewusstsein. Die mit einer gewissen Selbstverständlichkeit schon ins Leben gehen. Aber das ist halt, wie sagt man, so eine Resilienz, die man vielleicht auch braucht, die haben vielleicht andere auch nicht, ja. Wenn der Sender morgen zusperrt, dann gehe ich halt kellnern, alles gut.

(Absolventin der Bildenden Kunst, Studienabschluss 2013)

Damit wird die Ambivalenz der Interviewten deutlich. Denn so sehr symbolisches Kapital als wichtiger Einflussfaktor gesehen wird, so sehr wird die soziale Herkunft aus einem weniger ausgestatteten Milieu als Ressource zur Umschiffung schwieriger Phasen der Existenzsicherung eingeschätzt. In Bezug auf die Akademie wird allerdings in den Interviews festgehalten, dass eine geringe soziale Durchlässigkeit gegeben sei.

Ich habe jetzt nicht das Gefühl, dass es so offen oder durchlässig war, sagen wir jetzt mal, für Personen mit einem Arbeiterklasse-Background.

(Absolvent*in der Bildenden Kunst, Studienabschluss 2017)

Von allen Studien, die ich gemacht habe, war die Akademie sicher der privilegierteste Ort, den ich mitbekommen habe. Und ich habe zum ersten Mal ärger selber irgendwie gecheckt, dass ich einen anderen Hintergrund habe.

(Absolventin des Masters in Critical Studies, Studienabschluss 2016)

Zurückgeführt wird dies unter anderem auch auf den Zugang zum Studium. Interviewte Absolvent_innen nehmen an, dass beispielsweise durch die Zulassungsverfahren größere Diversität unter den Studierenden eher behindert als gefördert werde. 


\subsection{Zwischenfazit}

Der Übergang vom Studium in die jeweiligen Berufs- und Tätigkeitsfelder gestaltet sich fließend. Vielfach waren die Absolvent_innen bereits während des Studiums in einem studienbezogenen Feld erwerbstätig und können daran anschließen. Erfolge, die für die berufliche Tätigkeit unterstützend wirken, stellen sich vor allem in der Lebenslage während oder kurz nach dem Studium ein. Diese Einschätzung der Befragten ist unabhängig davon, wie lange ihr Studienabschluss zurückliegt. Die Gründe für Brüche in der Berufstätigkeit liegen laut Selbstwahrnehmung in den prekären Rahmenbedingungen des künstlerischen Umfelds, die auch immer wieder zu Phasen der Arbeitslosigkeit führen kann. Frauen thematisieren Familiengründungen und die Vereinbarung von Beruf und Privatem vermehrt in Zusammenhang mit Brüchen in der Erwerbsbiografie. Dies spiegelt sich auch in dem statistischen Modell zu Faktoren der Erwerbsintegration wider, das hauptsächlich auf strukturelle Einflüsse für die dauerhafte Integration in ein studienbezogenes Berufsfeld verweist.

Erwerbsprekarität stellt aus Sicht der befragten Absolvent_innen eine zentrale Herausforderung für die berufliche Integration dar. Als weitere wichtige Faktoren zeigen die Ergebnisse Probleme bei der sozialen Absicherung, die Vereinbarkeit von Privatem und Beruf sowie Selbstzweifel. Die Schwierigkeiten der sozialen Absicherung ergeben sich zu einem nicht unbeträchtlichen Teil aus der prekären Erwerbsarbeit.

Diskriminierung wird sowohl im künstlerischen Umfeld als auch an der Akademie thematisiert. Eine hohe Sensibilität in Fragen von Ungleichbehandlungen wurde der Akademie vor allen in Hinblick auf die Diskriminierungsgründe Geschlecht und Herkunft zugestanden. Kritisch beurteilen die Absolvent_innen die soziale Durchlässigkeit an der Akademie. Insbesondere in den Interviews werden Momente thematisiert, die als Ausschluss auf Grund der sozialen Herkunft empfunden wurden. 


\section{DAS STUDIUM AN DER AKADEMIE}

\subsection{Reflexion des Studiums}

Rund 37\% der befragten Absolvent_innen der Akademie haben ein weiteres Studium absolviert, für die Studienrichtung Bildende Kunst liegt dieser Anteil bei $61 \%$. Rund 34\% verfügen zusätzlich zum Studium über eine nicht-akademische Berufsausbildung. Der überwiegende Teil von innen (70\%) hat eine berufsbildende Schule oder Lehre im künstlerischhandwerklichen Bereich (z.B. Fotografie, Mediendesign, Grafik, Bildhauerei, Tischlerei) abgeschlossen.

Die Zeit des Studiums an der Akademie wird im Rückblick der Befragten als Freiraum erlebt. Insbesondere in den Interviews wird deutlich, dass die Ermöglichung künstlerischer Arbeit ohne ökonomischen Verwertungszwang als wichtiges individuelles Entwicklungsfeld angesehen wird. Durch die Verfügbarkeit von Arbeitsräumen, Geräten und Arbeitsmaterial werden laut den Erhebungsergebnissen gute und unterstützende Rahmenbedingungen für die Studierenden geschaffen.

Neben der grundsätzlich positiven Bewertung der, vergleichsweise zu anderen Studien freien Organisationsstruktur wird genau diese Freiheit auch als herausfordernd reflektiert.

Mit so viel Freiheit überhaupt umzugehen, gerade auch weil man idealerweise dabei ist sich selber eine künstlerische Strategie, Handlungsweise und so weiter anzueignen und zu entwickeln, und gleichzeitig, halt das Studium zu managen. Teilweise wenn man sich's genauer anschaut, kommt man vielleicht drauf, dass sich des a bissl spießt und widerspricht. (Absolvent der Bildenden Kunst, Studienabschluss 2016)

Ich habe immer so ein bisschen geschmunzelt über die Leute, die halt so super organisiert waren, weil ich's jetzt nicht war. Und jetzt muss ich's halt lernen, weil sonst geht's nicht. (Absolventin des Künstlerischen Lehramts, Studienabschluss 2019)

Der theoretische und gesellschaftspolitische Diskurs an der Akademie, der nach Einschätzung der Interviewten von kapitalismuskritischem Denken und einem kritischen Kunstbegriff geprägt ist, wird einerseits als sehr herausfordernd geschätzt. Anderseits schaffe er auch Widersprüchlichkeiten und Ausschlüsse. Die Ambivalenz zwischen dem als positiv angesehenen Freiraum sowie dem gesellschaftskritischen Diskurs während des Studiums und den Herausforderungen, sich auf einem Markt innerhalb einer kapitalistischen Logik zu bewegen, ist ein wiederkehrendes Thema in den Interviews.

Ja, weil das Level auf der Akademie, also was für mich halt wichtig war, der Diskurs und die theoretische Auseinandersetzung einfach sehr hoch ist und sehr abstrakt. Und das war, also, sich ein Bild zu machen, dass man da wirklich reinpasst. Und da habe ich eben nicht so wirklich das Leiberl dafür gehabt. (Absolventin der Bildenden Kunst, Studienabschluss 2016)

Es ist schön - und das find ich auch richtig - große Verwertungszusammenhänge kritisch zu sehen, aber es geht schon auch drum, wie die Leute zu ihrem Geld kommen, am Anfang oder am Ende des Monats halt. Und man muss das auch sagen können. Also, nicht über solche Sachen zu sprechen, das trifft für mich nur Privilegien - und Privilegien, die ich jetzt einfach nicht hab. Also, wenn die Leute nicht wissen, wie sie mit ihrer Kunst zu Geld kommen können oder was sie fordern sollen und so weiter und so fort - dann ist das halt schwierig. (Absolventin des Masters in Critical Studies, Studienabschluss 2016) 
Die Akademie wird als übersichtlich angesehen. Die Befragten bewerten das Betreuungsverhältnis zwischen Lehrkräften und Studierenden als hervorragend. Eine enge und intensive Zusammenarbeit in der künstlerischen und fachlichen Praxis und Entwicklung werde dadurch ermöglicht. Einige Interviewte streichen hervor, dass insbesondere zu den Lehrenden des Mittelbaus ein Naheverhältnis bestehe. Ihnen kommt demnach eine wichtige Rolle bei der Unterstützung und Begleitung der Studierenden zu.

Und dann hat man sehr gute Assistentinnen, die ja auch meistens Künstlerinnen und Künstler sind, nur ein bisschen jünger, oder eben Techniker und sich gut auskennen vielleicht, die auf jeden Fall immer da waren, weil natürlich haben die Professoren auch mal ihre eigene Ausstellung und sind auch nicht jede Woche da, aber sind immer ansprechbar, man hatte immer die Möglichkeit Gesprächstermine zu finden, also da habe ich mich schon sehr zu Hause gefühlt.

(Absolventin der Bildenden Kunst, Studienabschluss 2011)

Gleichzeitig wird das enge Betreuungsverhältnis auch in Bezug auf mögliche Abhängigkeiten reflektiert. Dabei wird deutlich, dass die Akademie in den letzten Jahren wichtige Schritte gesetzt hat. Ältere Absolvent_innen erzählen von teilweise feudalen Abhängigkeiten und willkürlich erscheinenden Beurteilungen aufgrund persönlicher Vorlieben. Auch von Sexismus und Diskriminierung ist die Rede. Hier sei ein Generationenwechsel auf der Ebene der Professor_innen feststellbar. Die „neue“ Generation wird als professionell beschrieben. Sie sei stärker theoretisch ausgerichtet und mache weniger Aufhebens um ihre Person.

Erwerbsarbeit mit dem Studium zu verbinden, wird auch kritisch gesehen. Aus den Interviews ergibt sich, dass die Studienorganisation teilweise schwierig war, da Studiengänge als Vollzeitstudien konzipiert sind und Zeiten von Lehrveranstaltungen mitunter mit Erwerbsarbeit kollidieren.

Aber es war ein wahnsinniger Stress und gegen Ende, wo mir nur mehr ein paar Lehrveranstaltungen gefehlt haben, hätte ich fast kündigen müssen, weil ich genau dieses Seminar machen musste, weil das war einfach das letzte das über war. Da habe ich keine Auswahl mehr gehabt. Und das hat sich nur mit einem Schulwechsel lösen lassen. (Absolventin des Künstlerischen Lehramts, Studienabschluss 2016) 


\subsection{Vorbereitung auf den Einstieg ins studienbezogene Feld}

Die Mehrzahl der Befragten fühlt sich durch ihr Studium sehr gut oder gut für den Einstieg in ein studienbezogenes Feld vorbereitet (43\%), ein weiterer Teil sieht dies zumindest teilweise so. Rund ein Fünftel (19\%) bewerten das Studium als eher nicht oder gar nicht hilfreich für den beruflichen Einstieg.

Abbildung 17

Einschätzung der Vorbereitung auf den Einstieg in ein studienbezogenes Feld in Prozent

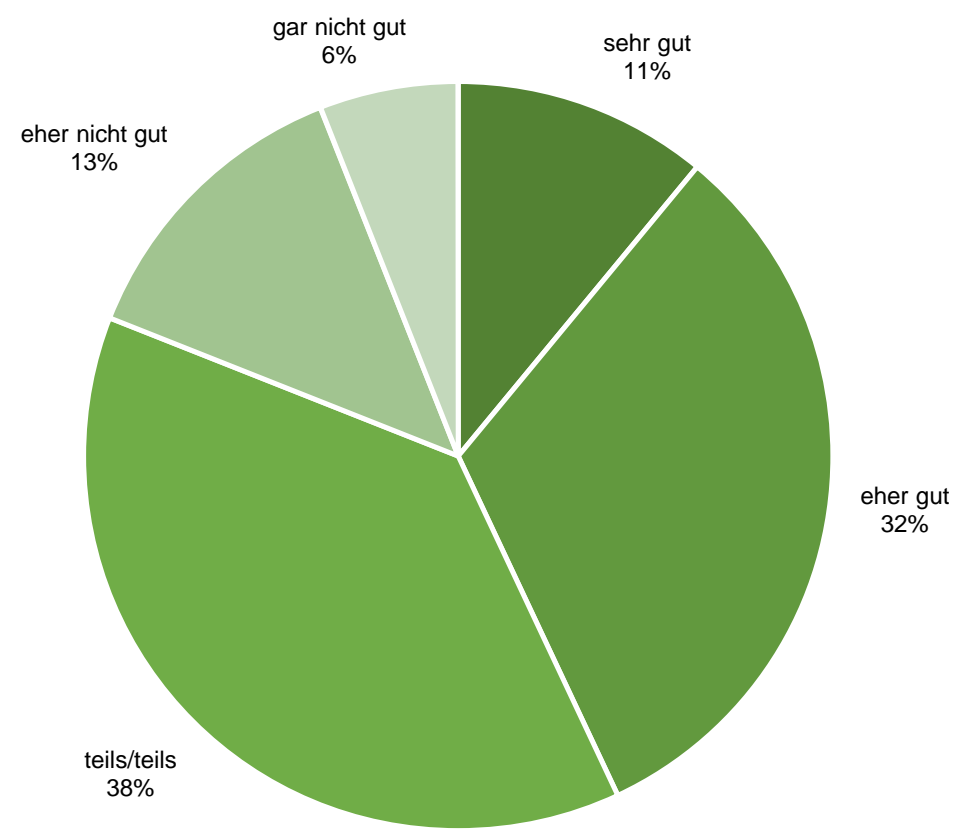

Quelle: ZSI - Absolvent_innenstudie 2000-2018

In diesem Zusammenhang tritt vor allem in den Interviews eine Reihe von kritischen Reflexionen hervor. Gleichzeitig gelingt es der Akademie offensichtlich gut, ihre Studierenden auf den Berufseinstieg vorzubereiten. Dies belegen die Ergebnisse zum Übergang vom Studium in die Erwerbsarbeit und der beruflichen Integration in einem studienbezogenen Feld (vgl. Kapitel 3 und 4). Die bisherigen Betrachtungen und Interpretationen legen den Schluss nahe, dass einige Kernbereiche für diesen Umstieg besonders wichtig sind. Neben den vermittelten Kompetenzen und Fähigkeiten, die in diesem Abschnitt betrachtet werden, ist dies laut Angabe der Befragten die Auseinandersetzung mit Kunst als Gegenstand. Darüber hinaus führt, wie bereits erwähnt, der angeführte Freiraum im Lauf des Studiums scheinbar zur Stärkung des Selbstbewusstseins und einer eigenständigen künstlerischen und beruflichen Positionierung. 


\subsection{Vermittelte Kompetenzen und Fähigkeiten}

Ein Abschluss einer Kunstuniversität hat aus Sicht der Befragten Bedeutung für den Übergang in die Beruflichkeit und für die Etablierung in einem studienbezogenen Feld. Das kann sich sowohl auf die formale Qualifikation als auch auf die vermittelten Kompetenzen beziehen. Daher wurde in der Befragung erhoben, welche Kompetenzen und Fähigkeiten aus Sicht der Absolvent_innen als wichtig für das Reüssieren im künstlerischen Umfeld angesehen werden. Darüber hinaus wurde die Einschätzung abgefragt, ob diese Kompetenzen und Fähigkeiten im Zuge des Studiums aus ihrer Sicht gefördert wurden.

Abbildung 18

Gegenüberstellung: Wichtigkeit der Kompetenzen im Feld mit Bezug zum Studium ${ }^{18}$ und Einschätzung der Förderung an der Akademie, Mittelwerte

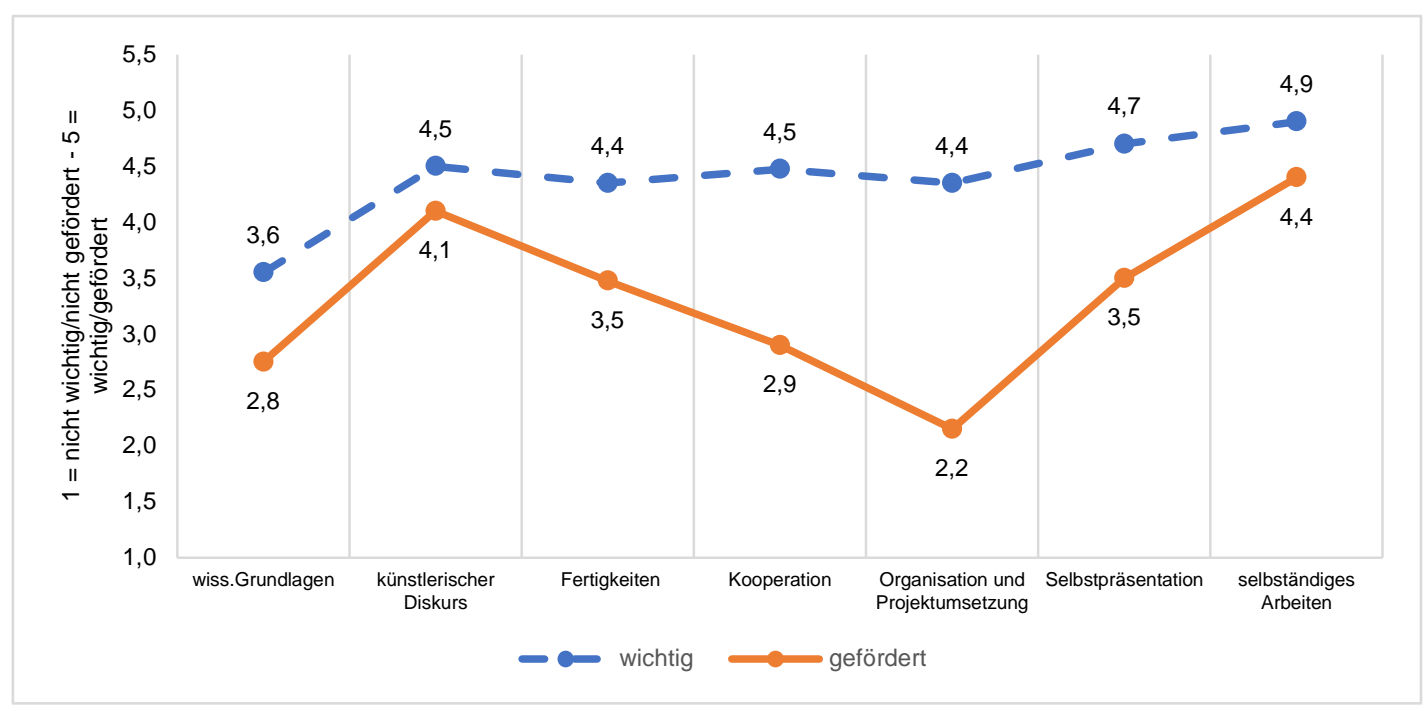

Quelle: ZSI - Absolvent_innenstudie 2000-2018, eigene Berechnungen

Nach Einschätzung der Befragten sind die Bereiche des künstlerischen und theoretischen Diskurses (kritisch-analytisches Denken und die Fähigkeit zur kritisch-analytischen Auseinandersetzung mit Kunst) und das Erlernen von selbständigem Arbeiten im Rahmen des Studiums gut abgedeckt. Verbesserungsbedarf in Bezug auf Studieninhalte wird bei Kompetenzen für Kommunikation und Kooperation gesehen. Unter diesen Schlagworten sind Netzwerkbildung, Teamarbeit und Kommunikationsfähigkeit sowie Sprachkenntnisse subsumiert. Insbesondere bei der Förderung für den Aufbau von Netzwerken regen die Befragten mehr Kompetenzvermittlung an. Diese werden im Mittelwert mit 4,6 als wichtige Fähigkeit angesehen, ihre Förderung während des Studiums hingegen mit vergleichsweise niedrig bewertet (Mittelwert 2,5).

${ }^{18}$ Vgl. Tabelle A5 für die Ausprägung der einzelnen Indikatoren. Folgende abgefragten Kompetenzen wurden für die Darstellung Gruppen zusammengefasst.

$\neg \quad$ wiss. Grundlagen: bildungswissenschaftliche Grundlagen (pädagogisch-didaktische Fähigkeiten), Kenntnisse wissenschaftlicher Methoden und Arbeitsweisen;

$\neg \quad$ künstlerischer Diskurs: Fähigkeit zu kritisch-theoretischen Auseinandersetzung mit Kunst, kritischesanalytisches Denken;

$\neg$ Fertigkeiten: künstlerischer Ausdruck, technisch-künstlerische Fähigkeiten, Fähigkeiten zur Vermittlung fachspezifischer Inhalte, Computerkenntnisse - computerunterstütztes Arbeiten;

$\neg \quad$ Kooperation: Team- und Kooperationsfähigkeit, Fähigkeit berufliche Netzwerke aufzubauen, Kommunikationsfähigkeit, Sprachkenntnisse;

$\neg \quad$ Organisation: unternehmerische Kenntnisse (Marketing, Rechtsgrundlagen, Steuern ...) Organisationsfähigkeit, Projektmanagement. 
Ein noch stärkerer Bedarf wird im Bereich von Organisation und Projektumsetzung gesehen, in denen unternehmerische Kenntnisse wie Steuerfragen oder Sozialversicherungsregelungen sowie Projektmanagement zusammengefasst wurde. Die befragten Absolvent_innen sehen die Vermittlung von unternehmerischen Kenntnissen wenig gefördert (Mittelwert 1,6), messen diesen aber eine vergleichsweise hohe Wichtigkeit zu (Mittelwert 4,1).

Aber es wäre nicht schlecht, wenn's auch ein bisschen weltlichere Vorlesungen gäbe. Es klingt jetzt einfach blöd, weil das einfach unromantisch ist, aber wenn man weiß, wie man seine Buchhaltung macht, bringt das einem Künstler schon ein bisschen mehr als alles andere. Wenn ich weiß, wie ich ein Kuratorengespräch führe, oder ein Verkaufsgespräch führe, oder Verhandlungen führe oder auch rechtliche Sachen.

(Absolventin der Bildenden Kunst, Studienabschluss 2011)

Damit kann festgehalten werden, dass die Auseinandersetzung und Vermittlung in künstlerischen Inhalten und Kompetenzen als gut und wichtig eingeschätzt werden. Kompetenzen und Fähigkeiten, die in dem wenig reglementierten und hochflexiblen, projektorientierten Arbeitsfeld, mit wechselnden Beschäftigungsformen und ausgeprägter selbstständiger Erwerbsarbeit über die künstlerische Kompetenz hinaus wichtig sind, sollten aus der reflektierenden Sicht der Absolvent_innen stärker gefördert werden.

Ein weiteres Ergebnis ist, dass es der Akademie gut gelingt, ihren Absolvent_innen Selbstkompetenz und Selbstbewusstsein in Bezug auf ihre studienbezogene und künstlerische Tätigkeit zu vermitteln. Die Interviewten erzählen, dass sie sich in ihrer individuellen beruflichen Positionierung, im Sinne der Auseinandersetzung mit dem eigenen Werk und der eigenen Kreativität nicht nur gut aufgehoben gefühlt haben, sondern auch eine gewisse Sicherheit sich selbst gegenüber erlangen konnten.

Und das ist das, was ich, glaube ich auf der Akademie gelernt habe, eine Story zu erzählen. So, was ist die Idee und was ist die Story des Gebäudes - sehr einfach erzählt. Und das hilft dir dann auch praktisch bis zum letzten Schrauben, das so quasi durchzukonstruieren. (Absolvent der Architektur, Studienabschluss 2001)

Also was nicht nur Bedienung ist, das zu erfüllen, was ein anderer erwartet, sondern halt etwas, wo man selbst weiß, man hat da was geleistet, hat was eingebracht, was auch für einen selber mehr Sinn gibt und schön ist, genau.

(Absolventin der Bildenden Kunst, Studienabschluss 2016)

Aber das kann man ja selber zum Glück mitgestalten und für mich ist es immer noch intellektuell extrem spannend, einfach, weil, ich kann sprachlich und nicht-sprachlich über Phänomene nachdenken. (Absolventin des Masters in Critical Studies, Studienabschluss 2016)

In der Selbstwahrnehmung der Befragten sind Kontakte aus Studienzeiten eine wichtige Ressource für berufliche Netzwerke nach dem Studium. Dabei sind Unterschiede zwischen Studienrichtungen erkennbar. Generell besteht ein intensiver Kontakt zu ehemaligen Studienkolleg_innen, der regelmäßig gepflegt wird. Besonders ausgeprägt ist dies in den Studienrichtungen Künstlerisches Lehramt und Architektur. Der Kontakt zu den Lehrenden gestaltet sich etwas unregelmäßiger, wird aber von über 50\% der Absolvent_innen gepflegt und als wichtig eingeschätzt. Aber auch Veranstaltungen, wie z.B. der Rundgang und Weiterbildungsangebote der Akademie werden besucht und zur Kontaktpflege genutzt. 


\subsection{Wirkung von Praktika und Mobilität}

Entgegen der bildungspolitischen Debatte, die Praktika einen förderlichen Stellenwert für den Berufseinstieg zuweist, ist in den Ergebnissen der Befragung kein derartiger Einfluss nachweisbar. Eine Begründung dafür kann der hohe Anteil jener Personen sein, die während des Studiums erwerbstätig ist und ohnehin Berufserfahrung sammelt. Davon abgesehen absolvieren rund 30\% freiwillige Praktika, dieser Anteil ist in den letzten Jahren deutlich gestiegen. Da bei weitem nicht alle Praktika bezahlt sind, ergibt sich hier eine besondere Herausforderung für erwerbstätige Studierende, sowohl in Hinblick auf Pflicht- als auch auf freiwillige Praktika.

Der Anteil der Frauen, die ein freiwilliges Praktikum absolvieren, liegt im Befragungssample bei einem Drittel (33\%) und damit höher als jene 25\% der befragten Männer mit freiwilligem Praktikum. Gleichzeitig geben Männer zu einem höheren Anteil als Frauen an, nur bezahlte Praktika absolviert zu haben (39\% zu 22\%). Es könnte sein, dass Männer eher auf ein Praktikum verzichten, wenn dieses nicht bezahlt ist. Eine derartige Einschätzung liegt zumindest in anderen Studien ${ }^{19}$ über Hochschulabsolvent_innen in Österreich vor.

Mobilität ist den Absolvent_innen insgesamt wichtig. Der Großteil der Absolvent_innen sammelt nach dem Studium internationale Erfahrungen und vernetzt sich. Dabei wird die Unterstützung der Akademie während des Studiums als sehr hilfreich angesehen. Auffallend ist, dass ehemalige Studierende im Befragungssample, die an einem ErasmusProgramm teilgenommen haben, auch nach ihrem Studium mobiler und international aktiver sind (vgl. Abbildung 19).

Abbildung 19

Internationalität nach dem Studium: Unterschiede in Bezug auf die Teilnahme an ErasmusProgrammen in Prozent

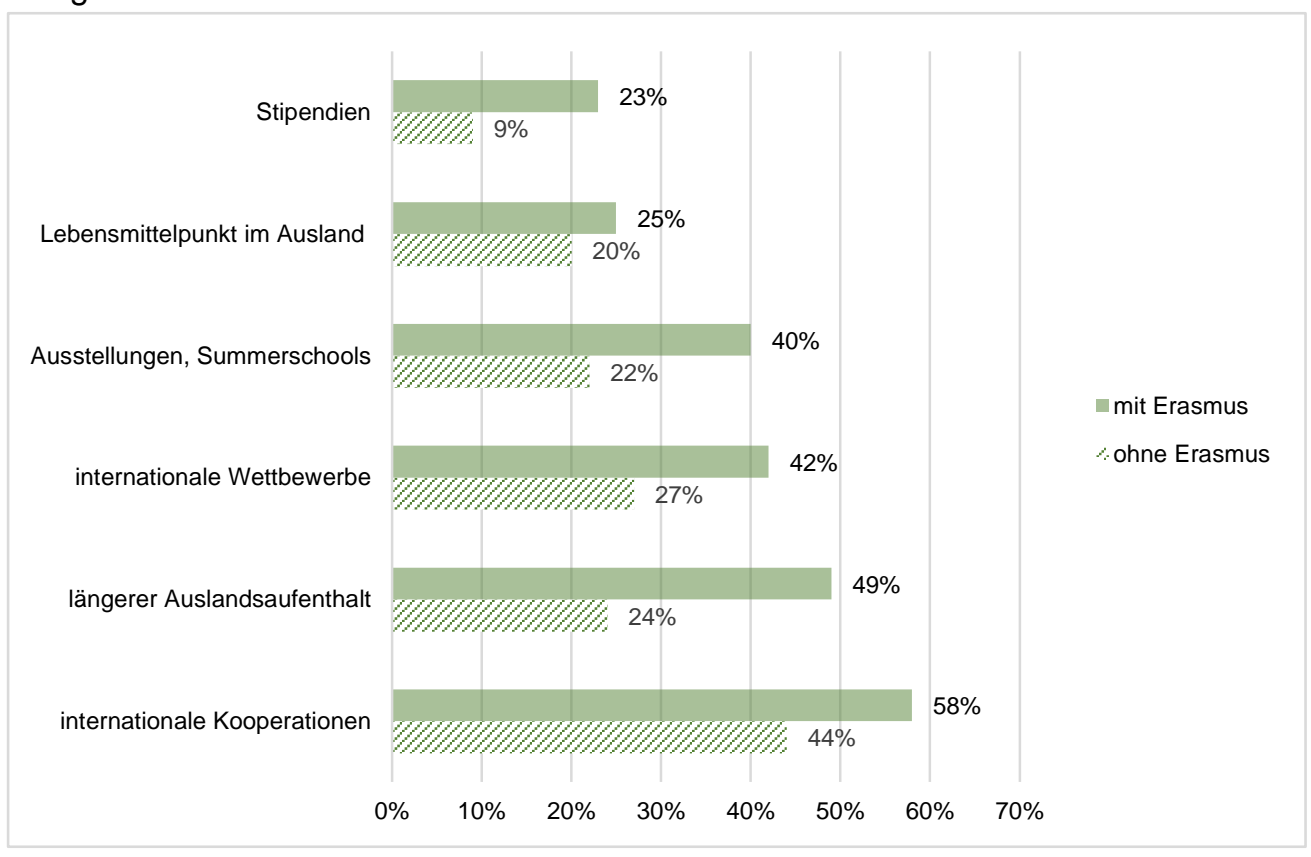

Quelle: ZSI - Absolvent_innenstudie 2000-2018

\footnotetext{
${ }^{19}$ Beispielsweise: Hubert Eichmann, Bernhard Saupe, (2011): Praktika und Praktikanten/Praktikantinnen in Österreich. Empirische Analyse von Praktika sowie der Situation von Praktikanten/Praktikantinnen. Forba-

Forschungsbericht 4. Online: https://www.forba.at/bericht/praktika-und-praktikanten-praktikantinnen-in-oesterreichempirische-analyse-von-praktika-sowie-der-situation-von-praktikanten-praktikantinnen/
} 
Während des Studiums sind mangelnde finanzielle Ressourcen das häufigste Mobilitätshindernis. Davon sind Frauen stärker betroffen als Männer (60\% zu 39\%). Insgesamt führen die Hälfte jener Befragten, die keinen Auslandsaufenthalt absolviert haben, dies auf finanzielle Gründe zurück (vgl. Tabelle 5). Ein weiteres Hindernis stellen mangelnde Sprachkenntnisse dar. Hier baut die Akademie unter anderem mit Lehrveranstaltungen in englischer Sprache vor, die nicht nur den internationalen Studierenden am Standort entgegenkommen, sondern auch Mobilität dahingehend unterstützen, dass künstlerischwissenschaftlicher Diskurs in einer Fremdsprache geübt und verbessert werden kann.

Frauen geben zu einem höheren Anteil als Männer an, dass die Unsicherheit bei der Rückkehr für sie ein Mobilitätshindernis darstellt (30\% zu 17\%). Entlang der bisherigen Befunde überrascht es, dass ein höherer Anteil an Männern als an Frauen Betreuungspflichten als Hindernis für einen Auslandsaufenthalt während des Studiums nennt. Das kann unterschiedliche Gründe haben, am wahrscheinlichsten ist, dass mehr Männern als Frauen in diesem Befragungs-Sample zu ihrer Studienzeit Betreuungspflichten bzw. Familie und Kinder hatten.

Tabelle 5

Mobilitätshindernisse in Bezug auf Auslandsaufenthalt während des Studiums nach Geschlecht (binär) in Prozent (Mehrfachantworten möglich)

\begin{tabular}{|c|c|c|c|}
\hline Mobilitätshindernisse & weiblich & männlich & gesamt \\
\hline mangelnde finanzielle Ressourcen & $60 \%$ & $39 \%$ & $52 \%$ \\
\hline kein Interesse & $23 \%$ & $39 \%$ & $29 \%$ \\
\hline Unsicherheit bei der Rückkehr & $30 \%$ & $17 \%$ & $26 \%$ \\
\hline Betreuungspflichten & $20 \%$ & $39 \%$ & $26 \%$ \\
\hline mangelnde Sprachkenntnisse & $23 \%$ & $22 \%$ & $22 \%$ \\
\hline mangelnde Fördermöglichkeiten & $23 \%$ & $13 \%$ & $18 \%$ \\
\hline Angst vor Verlust von Netzwerken & $15 \%$ & $17 \%$ & $15 \%$ \\
\hline steuer- und sozialrechtliche Fragen & $15 \%$ & $17 \%$ & $15 \%$ \\
\hline aufenthalts- und sozialrechtliche Fragen & $15 \%$ & $9 \%$ & $12 \%$ \\
\hline keine Anerkennung meiner Bildungsabschlüsse & $8 \%$ & $0 \%$ & $5 \%$ \\
\hline zu viele Verpflichtungen vor Ort & $3 \%$ & $4 \%$ & $3 \%$ \\
\hline persönliche Probleme & $3 \%$ & $0 \%$ & $2 \%$ \\
\hline
\end{tabular}

Quelle: ZSI - Absolvent_innenstudie 2000-2018

Viele Gründe für die Verhinderung der Mobilität liegen außerhalb des Einflussbereichs der Akademie. In den letzten Jahren wurde das Beratungs- und Unterstützungsangebot in diesem Bereich ausgebaut. Die Ergebnisse dieser Maßnahmen können sich zum jetzigen Zeitpunkt in dieser Befragung der Absolvent_innen noch nicht widerspiegeln. 


\subsection{Unterstützungsangebote der Akademie}

Die Akademie hat eine Reihe von Unterstützungsangebote für ihre Studierenden. Nichts desto trotz kann nach Ansicht der Befragten hier ausgebaut werden (vgl. Abbildung 20). Insbesondere bei Projektförderungen und der Vorbereitung für Wettbewerbe wird ein Bedarf moniert. Kontakte für den Berufseinstieg sind ebenso nachgefragt, wie jene zu Praktikumsmöglichkeiten und allgemeiner Vernetzung. Das heißt nicht unbedingt, dass die bestehenden Angebote der Akademie in diesen Punkten als nicht ausreichend oder mangelhaft eingestuft werden. Denn der Wunsch nach mehr Unterstützung kann auch als ein Zeichen von Vertrauen und Anerkennung gewertet werden. Jedenfalls sehen die Befragten im Rückblick auf ihr Studium ein Verbesserungspotential. Möglicherweise spiegeln diese Einschätzungen gleichzeitig Unsicherheiten der Absolvent_innen (in den abgefragten Kategorien) wider, die sie während ihrer Studienzeit empfunden haben.

Abbildung 20

Wunsch nach mehr Angeboten in ausgewählten Unterstützungsbereichen ${ }^{20}$ der Akademie in Prozent

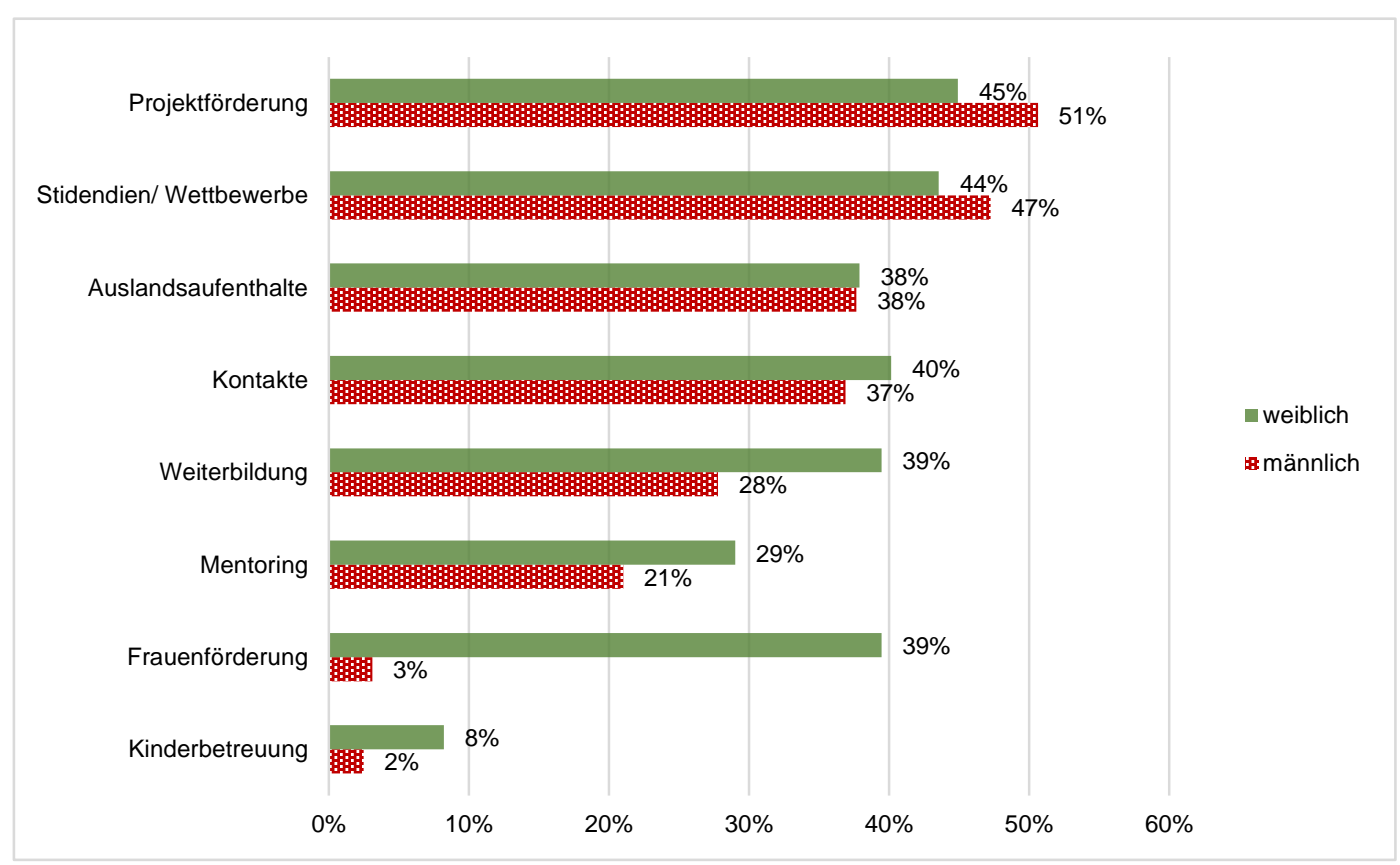

Quelle: ZSI - Absolvent_innenstudie 2000-2018, eigene Berechnungen

Bei diesen Ergebnissen ist zu beachten, dass sie nicht den aktuellen Ausbau an Unterstützungsangeboten an der Akademie anzeigen können. Sie sind aber wichtige Hinweise auf mögliche Bedarfe aus Sicht von Absolvent_innen.

\footnotetext{
${ }^{20} \mathrm{Vgl.}$ Tabelle A6 für die Ausprägung der einzelnen Indikatoren. Folgende Indikatoren wurden zusammengefasst: $\neg \quad$ Kontakt: Kontakte zu Arbeitgeber_innen, Praktika-Vermittlung, Vernetzungsmöglichkeiten;

$\neg \quad$ Auslandsaufenthalte: Unterstützung bei Auslandsaufenthalten/Erasmus, Unterstützung bei Auslandsateliers.
} 


\subsection{Zwischenfazit}

Die Absolvent_innen empfinden die Zeit des Studiums als Freiraum, der eine große Bandbreite an Entwicklungsmöglichkeiten mit sich bringt. Der vergleichsweise geringe Grad an formalisierter Organisation ruft aber auch Ambivalenzen hervor. Manche fühlen sich davon überfordert und wünschen sich ein Mehr an Struktur.

Hervorgehoben wird in den Interviews vor allem der breite Raum für gesellschaftskritischen und antikapitalistischen Diskurs, der als befruchtend angesehen wird. Allerdings thematisieren einige Interviewte auch eine gewisse Realitätsferne im Diskurs. So würde zu wenig auf die Anforderungen und Schwierigkeiten in einem kapitalistischen Kunstmarkt eingegangen. Die Notwendigkeit von Erwerbsarbeit und die Verwertungszwänge der eigenen künstlerischen Arbeit, um den Lebensunterhalt zu bestreiten, sowie die damit verbundenen Widersprüchlichkeiten in Bezug auf eine antikapitalistische Grundhaltung würden im Diskurs zu wenig Beachtung finden.

Die gemeinsame Arbeit mit Studienkolleg_innen im Rahmen des künstlerischen Unterrichts ist für die befragten Absolvent_innen eine wichtige Ressource für die künstlerische und persönliche Entwicklung. Das enge Betreuungsverhältnis wird generell positiv eingeschätzt, auch wenn die Nachteile von dadurch entstehenden Abhängigkeitsverhältnissen in den Interviews durchaus reflektiert werden. Befragte früherer Abschlusskohorten erachten in diesem Zusammenhang die Auflösung der so genannten Meisterklassen als positiv. Diese strukturelle Veränderung habe ein Mehr an Durchlässigkeit geschaffen und das Abhängigkeitsverhältnis zwischen Professor_in und der_dem einzelnen Studierenden deutlich entschärft.

Der Ausbildung an der Akademie bzw. den vermittelten Qualifikationen und Kompetenzen wird hohe Bedeutung für die berufliche Integration beigemessen. Das gilt vor allem in künstlerischen Fertigkeiten, künstlerisch-theoretischen Diskursfähigkeiten sowie in Fragen der Selbsteinschätzung und Positionierung im künstlerischen Umfeld bzw. studienbezogenen Feld. Ausbaufähig sind hingegen die Vermittlung von Kompetenzen im Bereich des Managements in Hinblick auf selbständige Erwerbsarbeit, der Projektumsetzung und damit verbundenen Know-how und gesetzlichen Regelungen. Kontakte aus der Studienzeit sind eine Ressource für berufliche Netzwerke und werden gepflegt. 


\section{RESÜMEE}

Der Akademie der bildenden Künste Wien gelingt es gut, ihre Studierenden bzw. Absolvent_innen auf die beruflichen Herausforderungen nach dem Studium vorzubereiten. Insbesondere in einem Feld, in dem die Arbeitsorganisation von Selbstmotivation und Selbstbewusstsein geprägt ist, hat die Akademie Mittel und Wege gefunden, dafür notwendige Kompetenzen und Selbstbilder während des Studiums zu vermitteln.

Diese Einschätzung wird unter anderem durch den hohen Anteil von Absolvent_innen, die sich selbst in ihrer Erwerbsarbeit im studienbezogenen Feld verorten, untermauert. Die Befragten fühlen sich durch das Studium in weiten Teilen auf die berufliche Tätigkeit vorbereitet. Dafür wurde innen eine Reihe von Kompetenzen und Fähigkeiten vermittelt. Einzig im Bereich der betriebswirtschaftlichen Kompetenzen und der Organisation von beruflicher Selbständigkeit ist diese Vermittlung im Studium aus Sicht der ehemaligen Studierenden nicht ausreichend.

Im Gesamtbild wird die Zeit des Studiums als positiv gesehen. Verbunden mit viel Freiraum und enthoben der Belastungen in Bezug auf Ausstattung mit Arbeitsmaterialien und Arbeitsraum, konnten sich die Befragten ihrem Studium widmen, hatten Raum für Entwicklung und Experimente und haben sich einen Pool von Ressourcen für ihre künstlerische Tätigkeit aufgebaut.

Viele Ansprüche und Herausforderungen, die von den Befragten durchaus kritisch gesehen werden, liegen nicht im Einflussbereich der Akademie bzw. des Studiums oder der Studienorganisation. Die größten Probleme bleiben die Prekarität in Bezug auf die Erwerbsarbeit und ein existenzsicherndes Einkommen. Damit eng verbunden sind die Ängste und Befürchtungen in Fragen der sozialen Absicherung. Wie der hohe Anteil an Erwerbsarbeit unter den Studierenden zeigt, begleitet sie dieses Thema von Anfang an.

Die gesellschaftliche Arbeitsteilung zwischen den Geschlechtern, die sich für Frauen in der Regel benachteiligend auswirkt, schlägt auch bei den Absolvent_innen durch. Die Auswirkungen lassen sich in Bezug auf das Einkommen und die Wochenarbeitszeiten in Erwerbsarbeit ebenso nachweisen, wie bei Belastungen durch Betreuungspflichten, Elternschaft und Reproduktionsarbeit allgemein. Hier wird von Seiten der Befragten hervorgehoben, dass an der Akademie der Diskurs über Gender breiten Raum bekommt, die Situation auBerhalb der Akademie allerdings eine andere ist.

Die Ergebnisse legen einige Punkte für die Debatte der Weitentwicklung an der Akademie nahe. Neben den inzwischen breit ausgebauten Maßnahmen über die Initiative Non-binaryUniversities ist die Frage nach einer besseren Durchmischung der Studierenden entlang sozioökonomischen Faktoren zu stellen. Über die Hälfte der Befragten kommt aus einem Herkunftshaushalt mit hohem ökonomischem Status.

Die Ergebnisse der qualitativen Erhebung zeigen, dass künstlerische Tätigkeit ein wichtiger Teil der Identität der Befragten ist. Auch im Fall von beruflichen Tätigkeiten, die eher am Rande des studienbezogenen Feldes verortet werden, wird die Verbindung mit künstlerischer Arbeit in den Interviews betont. Dafür werden von Absolvent_innen häufig schwierige Rahmenbedingungen in Kauf genommen.

Die Anforderungen des künstlerischen Umfelds werden kritisch hinterfragt. Dies gilt im Besonderen für die fließenden Übergänge zwischen Erwerbsarbeit und Freizeit in der künstlerischen Tätigkeit. Das permanente Selbstmarketing und die Notwendigkeit einer dauerhaften Präsenz in Netzwerken oder bei Veranstaltungen werden zum Teil als Hinderungsgrund 
für den weiteren Ausbau der künstlerischen Tätigkeit empfunden. Gleichzeitig werden sie als „ein Mythos“ angesehen, wie die Interviews zeigen. Tragfähige Netzwerke sind für alle Befragten wichtige Ressourcen, doch ergeben sie sich eher durch gemeinsame Projekte oder andere Formen der gemeinsamen künstlerischen Arbeit als durch die Präsenz bei Events. Bei diesen Widersprüchlichkeiten ist auffällig, das Interviewte, die sich durch Betreuungspflichten in den Möglichkeiten der Teilnahme an Events beschränkt sehen, das eher als einen Hinderungsgrund für berufliches Vorankommen empfinden, jene, die hier weniger Verpflichtungen aufweisen, diese Events als überbewertet betrachten.

Die Vermittlung von spezifischen Kompetenzen für das künstlerische Arbeiten, wird sowohl im künstlerisch-praktischen, im theoretisch-diskursiven Bereich als auch bei der generellen Unterstützung der künstlerischen Entwicklung gut abgedeckt.

Die Zulassungsverfahren führen nach Ansicht der Befragten dazu, dass die Diversität in Hinblick auf den sozialen Status eingeschränkt ist. Eine soziale Durchlässigkeit sei gering verwirklicht.

Im künstlerischen Umfeld spielt symbolisches Kapital im Sinne Bourdieus eine wichtige Rolle bei der Integration. Eine ungleiche Verteilung damit kann die Akademie im Rahmen des Studiums nicht ausgleichen, sondern allenfalls dämpfen. Dies spiegelt sich auch in dem teilweise als abstrakt empfundenen gesellschaftstheoretischen Diskurs auf hohem Niveau, wie er an der Akademie geführt wird, wider. Das führt, wie die Interviews zeigen, mitunter zu Ausschließungsmechanismen. 


\section{ANHANG}

\subsection{Indikatoren im Einzelnen}

Tabelle A1

Einzelne Indikatoren zu Abbildung 7: Wichtige Faktoren der Arbeitszufriedenheit und deren Erfüllungsgrad in Prozent

Fragen aus dem Fragebogen: Wie wichtig sind folgende Faktoren bei Ihrer Arbeit? - Und in wieweit sehen Sie diese Faktoren in Ihrer derzeitigen Arbeitssituation erfüllt?

\section{Faktoren der Zufriedenheit}

Freude an der Arbeit

kreativ sein

existenzsicherndes Einkommen

sinnvolle Arbeit

gutes Arbeitsklima

persönliche Entwicklungsmöglichkeiten

Anerkennung meiner Arbeit

Vereinbarkeit von Privatem und Beruf

gesellschaftlich wirksam sein

abwechslungsreiche Tätigkeit

gute Ausstattung mit Arbeitsmitteln

meine im Studium erworbenen Qualifikationen einsetzen können

Anerkennung meiner Arbeit

Teamarbeit

geregelte Arbeitszeiten

hohes Einkommen

Quelle: ZSI - Absolvent_innenstudie 2000-2018
Anteil Anteil wichtig wichtig und erfüllt

97\% $\quad 76 \%$

$96 \% \quad 70 \%$

$96 \% \quad 53 \%$

$96 \% \quad 72 \%$

$94 \% \quad 70 \%$

$94 \% \quad 54 \%$

$93 \% \quad 52 \%$

$89 \% \quad 54 \%$

$88 \% \quad 53 \%$

$88 \% \quad 80 \%$

$83 \% \quad 48 \%$

$68 \% \quad 59 \%$

$67 \% \quad 56 \%$

$62 \% \quad 69 \%$

$45 \% \quad 63 \%$

$44 \% \quad 25 \%$ 
Tabelle A2

Einzelne Indikatoren zu Abbildung 11: Einflussgrößen in Bezug auf die Integration in Erwerbstätigkeit in einem studienbezogenen Feld nach eingeschätzter Einflussgröße, Mittelwerte

Frage aus dem Fragebogen: Was ist wichtig, um sich in einem Berufsfeld mit Bezug zu Ihren Studium zu etablieren? Bitte wählen Sie die zutreffende Antwort für jeden Punkt aus.

\begin{tabular}{|c|c|}
\hline wichtige Elemente & Mittelwerte \\
\hline Durchhaltevermögen & 4,1 \\
\hline persönliches Auftreten & 3,4 \\
\hline Qualität der Arbeit & 3,3 \\
\hline sich selbst gut verkaufen können & 3,2 \\
\hline soziale Kompetenzen & 3,0 \\
\hline der Abschluss einer Kunsthochschule/Kunstuniversität & 2,8 \\
\hline frei von finanziellen Sorgen sein & 2,5 \\
\hline Preise/Stipendien & 2,5 \\
\hline Pflege von informellen Netzwerken & 2,4 \\
\hline von den richtigen Menschen "entdeckt zu werden" & 2,4 \\
\hline Kontakt zu Vermittler_innen und Veranstalter_innen & 2,3 \\
\hline aktives Marketing/Öffentlichkeitsarbeit & 2,3 \\
\hline Beteiligung an Wettbewerben, Ausschreibungen & 2,3 \\
\hline Talent & 2,3 \\
\hline gut deutsch zu sprechen & 2,3 \\
\hline Kontakt zu privaten Sammler_innen und Sponsor_innen & 2,2 \\
\hline das Ansehen der Akademie & 2,0 \\
\hline die_der Professor_in, bei der_dem ich studiert habe & 2,0 \\
\hline Arbeitsmittelpunkt im urbanen Raum & 1,9 \\
\hline Internetplattformen aktiv zu nutzen & 1,7 \\
\hline Teilnahme/Beteiligung an formellen Netzwerken & 1,7 \\
\hline den Geschmack des Publikums treffen & 1,7 \\
\hline
\end{tabular}

Quelle: ZSI - Absolvent_innenstudie 2000-2018 
Tabelle A3

Einzelne Indikatoren zu Abbildungen 13 und 14: Generelle Betroffenheit nach Geschlecht in Prozent, Häufigkeit der Diskriminierungserfahrungen nach Geschlecht, Mittelwerte

Frage aus dem Fragebogen: Haben Sie bei Ihrer Arbeit bzw. bei der Suche nach Arbeit bisher Situationen erlebt, in denen Sie Sich aufgrund folgender Faktoren ungerecht behandelt fühlten?

\begin{tabular}{|c|c|c|c|c|c|c|}
\hline $\begin{array}{l}\text { Auswertung } \\
\text { nach Ge- } \\
\text { schlecht }\end{array}$ & $\begin{array}{r}\text { weil } \\
\text { Anteil } \\
\text { Betroffen- } \\
\text { heit }\end{array}$ & $\begin{array}{l}\text { lich } \\
\text { Häufigkeit } \\
\text { Mittelwert }\end{array}$ & $\begin{array}{r}\text { mänı } \\
\text { Anteil } \\
\begin{array}{r}\text { Betroffen- } \\
\text { heit }\end{array}\end{array}$ & $\begin{array}{l}\text { lich } \\
\text { Häufigkeit } \\
\text { Mittelwert }\end{array}$ & $\begin{array}{r}\text { div } \\
\text { Anteil } \\
\text { Betroffen- } \\
\text { heit }\end{array}$ & $\begin{array}{l}\text { Häufigkeit } \\
\text { Mittelwert }\end{array}$ \\
\hline Geschlecht & $71 \%$ & 2,6 & $21 \%$ & 2,3 & $86 \%$ & 2,7 \\
\hline zu alt & $35 \%$ & 2,4 & $24 \%$ & 2,4 & $17 \%$ & 4,0 \\
\hline zu jung & $36 \%$ & 2,2 & $17 \%$ & 2,1 & $33 \%$ & 2,0 \\
\hline $\begin{array}{l}\text { persönliches } \\
\text { Auftreten }\end{array}$ & $45 \%$ & 2,4 & $34 \%$ & 2,3 & $80 \%$ & 2,5 \\
\hline Sprache & $19 \%$ & 2,5 & $12 \%$ & 2,4 & $50 \%$ & 2,7 \\
\hline $\begin{array}{l}\text { ethnische } \\
\text { Zugehörigkeit }\end{array}$ & $6 \%$ & 2,6 & $4 \%$ & 2,3 & $8 \%$ & 3,0 \\
\hline $\begin{array}{l}\text { kulturelle/ } \\
\text { religiöse } \\
\text { Zugehörigkeit }\end{array}$ & $6 \%$ & 2,5 & $4 \%$ & 2,4 & $8 \%$ & 3,0 \\
\hline $\begin{array}{l}\text { der Ort, an dem } \\
\text { ich geboren bin }\end{array}$ & $7 \%$ & 2,6 & $7 \%$ & 2,5 & $17 \%$ & 2,5 \\
\hline $\begin{array}{l}\text { sexuelle } \\
\text { Orientierung }\end{array}$ & $8 \%$ & 2,3 & $10 \%$ & 2,1 & $83 \%$ & 2,2 \\
\hline $\begin{array}{l}\text { körperliche } \\
\text { und/oder } \\
\text { psychische } \\
\text { Beeinträchtigung }\end{array}$ & $12 \%$ & 2,2 & $3 \%$ & 2,0 & $33 \%$ & 3,0 \\
\hline $\begin{array}{l}\text { sozioökonomi- } \\
\text { sche Situation }\end{array}$ & $28 \%$ & 2,6 & $23 \%$ & 2,3 & $62 \%$ & 2,4 \\
\hline $\begin{array}{l}\text { meine familiäre } \\
\text { Situation bzw. } \\
\text { Verpflichtungen }\end{array}$ & $34 \%$ & 2,9 & $20 \%$ & 2,5 & $0 \%$ & 0 \\
\hline
\end{tabular}


Tabelle A4

Einzelne Indikatoren zu Abbildungen 15 und 16: Generelle Betroffenheit nach Herkunft in Prozent, Häufigkeit der Diskriminierungserfahrungen nach Herkunft, Mittelwerte

Frage aus dem Fragebogen: Haben Sie bei Ihrer Arbeit bzw. bei der Suche nach Arbeit bisher Situationen erlebt, in denen Sie Sich aufgrund folgender Faktoren ungerecht behandelt fühlten?

\begin{tabular}{|c|c|c|c|c|c|c|}
\hline \multirow[b]{2}{*}{$\begin{array}{l}\text { Auswertung } \\
\text { nach Herkunft }\end{array}$} & \multicolumn{2}{|c|}{ Österreich } & \multicolumn{2}{|c|}{ Nicht-EU-Staaten } & \multicolumn{2}{|c|}{ EU } \\
\hline & $\begin{array}{r}\text { Anteil } \\
\text { Betroffen- } \\
\text { heit }\end{array}$ & $\begin{array}{l}\text { Häufigkeit } \\
\text { Mittelwert }\end{array}$ & $\begin{array}{r}\text { Anteil } \\
\text { Betroffen- } \\
\text { heit }\end{array}$ & $\begin{array}{c}\text { Häufigkeit } \\
\text { Mittelwert }\end{array}$ & $\begin{array}{r}\text { Anteil } \\
\text { Betroffen- } \\
\text { heit }\end{array}$ & $\begin{array}{l}\text { Häufigkeit } \\
\text { Mittelwert }\end{array}$ \\
\hline Geschlecht & $56 \%$ & 2,5 & $50 \%$ & 2,8 & $58 \%$ & 2,5 \\
\hline zu alt & $30 \%$ & 2,4 & $40 \%$ & 2,8 & $27 \%$ & 2,2 \\
\hline zu jung & $31 \%$ & 2,3 & $21 \%$ & 2,0 & $32 \%$ & 2,3 \\
\hline $\begin{array}{l}\text { persönliches } \\
\text { Auftreten }\end{array}$ & $41 \%$ & 2,4 & $41 \%$ & 2,7 & $51 \%$ & 2,3 \\
\hline Sprache & $13 \%$ & 2,3 & $44 \%$ & 2,7 & $19 \%$ & 2,5 \\
\hline $\begin{array}{l}\text { ethnische } \\
\text { Zugehörigkeit }\end{array}$ & $1 \%$ & 2,0 & $42 \%$ & 2,8 & $15 \%$ & 2,4 \\
\hline $\begin{array}{l}\text { kulturel- } \\
\text { le/religiöse } \\
\text { Zugehörigkeit }\end{array}$ & $4 \%$ & 2,0 & $24 \%$ & 3,1 & $10 \%$ & 2,4 \\
\hline $\begin{array}{l}\text { der Ort, an dem } \\
\text { ich geboren bin }\end{array}$ & $8 \%$ & 2,3 & $40 \%$ & 3,4 & $25 \%$ & 2,4 \\
\hline $\begin{array}{l}\text { sexuelle } \\
\text { Orientierung }\end{array}$ & $10 \%$ & 2,4 & $3 \%$ & 2,0 & $13 \%$ & 2,3 \\
\hline $\begin{array}{l}\text { körperliche } \\
\text { und/oder } \\
\text { psychische } \\
\text { Beeinträchtigung }\end{array}$ & $11 \%$ & 2,2 & $0 \%$ & 0 & $8 \%$ & 2,6 \\
\hline $\begin{array}{l}\text { sozioökonomi- } \\
\text { sche Situation }\end{array}$ & $28 \%$ & 2,5 & $20 \%$ & 3,2 & $29 \%$ & 2,3 \\
\hline $\begin{array}{l}\text { meine familiäre } \\
\text { Situation bzw. } \\
\text { Verpflichtungen }\end{array}$ & $29 \%$ & 2,9 & $21 \%$ & 3,3 & $33 \%$ & 2,4 \\
\hline
\end{tabular}


Tabelle A5

Einzelne Indikatoren zu Abbildung 18: Gegenüberstellung: Wichtigkeit der Kompetenzen im Feld mit Bezug zum Studium und Einschätzung der Förderung an der Akademie, Mittelwerte

Fragen aus dem Fragebogen: Wie sehr wurden die folgenden Fähigkeiten im Rahmen Ihres Studiums an der Akademie gefördert? - Wie wichtig sind die zuvor genannten Fähigkeiten, um in einem Feld mit Bezug zu Ihrem Studium tätig zu sein?

\section{Fähigkeiten}

gefördert wichtig Abweichung

unternehmerische Kenntnisse

(Marketing, Rechtsgrundlagen, Steuern ...)

Fähigkeit, berufliche Netzwerke aufzubauen

Organisationsfähigkeit/Projektmanagement

Sprachkenntnisse

Kommunikationsfähigkeit

Selbstpräsentation

Team- und Kooperationsfähigkeit

Computerkenntnisse, computerunterstütztes Arbeiten

technisch-künstlerische Fähigkeiten

bildungswissenschaftliche Grundlagen

(pädagogisch-didaktische Fähigkeiten)

Fähigkeit zur Vermittlung fachspezifischer Inhalte

Kenntnisse wissenschaftlicher Methoden und Arbeitsweisen

\begin{tabular}{rr|r}
1,6 & 4,1 & $-2,5$ \\
2,5 & 4,6 & $-2,1$ \\
2,7 & 4,6 & $-1,9$ \\
2,4 & 4,1 & $-1,7$ \\
3,4 & 4,8 & $-1,4$ \\
3,5 & 4,7 & $-1,2$ \\
3,3 & 4,4 & $-1,1$ \\
3,3 & 4,3 & $-1,0$ \\
3,5 & 4,5 & $-1,0$ \\
2,5 & 3,4 & $-0,9$ \\
3,4 & 4,3 & $-0,9$ \\
3 & 3,7 & $-0,7$ \\
3,7 & 4,3 & $-0,6$ \\
4 & 4,6 & $-0,6$ \\
4,4 & 4,9 & $-0,5$ \\
4,2 & 4,4 & $-0,2$
\end{tabular}

künstlerischer Ausdruck

kritisch-analytisches Denken

selbstständiges Arbeiten

Fähigkeit zur kritisch-theoretischen Auseinandersetzung

mit Kunst

Quelle: ZSI - Absolvent_innenstudie 2000-2018 
Tabelle A6

Einzelne Indikatoren zu Abbildung 20 Wichtigkeit der Unterstützungsangebote in Mittelwerten und Wunsch nach mehr Angebote der Akademie in Prozent

Fragen aus dem Fragebogen: Wie wichtig waren die folgenden Unterstützungsangebote der Akademie für Ihren Berufseinstieg? - Wobei hätten Sie gerne mehr Unterstützung durch die Akademie oder Angehörigen der Akademie (z.B. Lehrende) gehabt?

Unterstützungsangebote

Förderung

praktische Hilfe durch Kontakte zu potentiellen

Auftraggeber_innen

Stipendien

Projektförderung

Vorbereitung für die Teilnahme an Wettbewerben

Vernetzungsmöglichkeiten (Alumnae_Alumniverein, ...)

Vermittlung von Auslandsresidenzen

("Artist in Residence", ...)

Vermittlung von Auslandsstudienplätzen

(Erasmus, ...)

Vortrags- und Ausstellungsmöglichkeiten

(xposit, ...)

Vermittlung von Praktikumsmöglichkeiten

Weiterbildungen

Teilnahme an Mentor_innenprogrammen

(ArtStart, ...)

Frauenförderung

Quelle: ZSI - Absolvent_innenstudie 2000-2018
Wichtigkeit in Mittelwerten

$57 \%$

$52 \%$

$49 \%$

$43 \%$

$42 \%$

$41 \%$

$39 \%$

$38 \%$

$38 \%$

$36 \%$

$28 \%$ 